\title{
Les perspectives de la recherche : patrimoine et architecture, urbanisme et paysages
}

Table ronde animée par Karen Bowie

Round table. Historical perspectives: Cultural heritage and architecture, urbanism and landscapes,

François Loyer, Evelyne Lohr, Anne Hecker et Jean-François Belhoste

\section{OpenEdition}

\section{Journals}

Édition électronique

URL : https://journals.openedition.org/rhcf/1028

DOI : 10.4000/rhcf.1028

\section{Éditeur}

Rails \& histoire

Édition imprimée

Date de publication : 1 juin 2008

Pagination : 255-292

ISSN : 0996-9403

Référence électronique

François Loyer, Evelyne Lohr, Anne Hecker et Jean-François Belhoste, «Les perspectives de la recherche : patrimoine et architecture, urbanisme et paysages », Revue d'histoire des chemins de fer [En ligne], 39 | 2008, mis en ligne le 01 juin 2011, consulté le 22 avril 2022. URL : http:// journals.openedition.org/rhcf/1028 ; DOI : https://doi.org/10.4000/rhcf.1028

Ce document a été généré automatiquement le 22 avril 2022

Tous droits réservés 


\section{Les perspectives de la recherche: patrimoine et architecture, urbanisme et paysages}

Table ronde animée par Karen Bowie

Round table. Historical perspectives: Cultural heritage and architecture, urbanism and landscapes,

François Loyer, Evelyne Lohr, Anne Hecker et Jean-François Belhoste

\section{NOTE DE L'ÉDITEUR}

La diffusion de certaines illustrations n'est pas autorisée sur l'Internet. Les lecteurs sont invités à consulter l'édition imprimée de la Revue d'histoire des chemins de fer pour prendre connaissance de l'article complet.

\section{Karen Bowie}

Je remercie Jean Fosseyeux d'avoir dressé un bilan précis et analytique de nos activités depuis vingt ans avant que nous consacrions un débat aux perspectives de la recherche dans le domaine du patrimoine architectural, urbain et paysager ferroviaire. Il doit permettre non seulement un échange de réflexions entre les intervenants, mais aussi avec tous les participants au colloque.

2 Afin de réfléchir ensemble aux "perspectives de la recherche: patrimoine et architecture, urbanisme et paysages", nous avons communiqué à chacun des intervenants l'appel à la recherche lancé par l'AHICF et élaboré par Jean Fosseyeux et moi-même en ce qui concerne le domaine « chemin de fer, patrimoine, architecture et paysage » dans lequel nous avons développé le thème : «Connaissance du patrimoine ferroviaire : architecture, urbanisme, paysage. » Nous les avons invités à se sentir libres d'y réagir ou non selon ce qu'il leur paraissait important d'exprimer. 


\section{François Loyer}

Je vais vous proposer quelques remarques en introduction. Elles sont très générales, assez éloignées de notre quotidien, mais nécessaires peut-être pour expliquer la raison d'être de l'activité de l'AHICF dans le domaine de l'architecture et du patrimoine. Je dirai d'abord la surprise d'un homme de ma génération de voir le chemin de fer survivre à la disparition qui lui était promise dans ma jeunesse. Je pensais en effet que, fils de marin, je ne verrais plus de bateaux et que, neveu de gens du chemin de fer, je ne verrais plus de trains, car l'autoroute et les avions, qui étaient alors pour moi le moyen du déplacement, les auraient remplacés définitivement. C'est le contraire qui s'est produit : la démocratisation de l'automobile qu'on nous avait annoncée a abouti à la congestion des transports et à de dramatiques problèmes d'environnement, tandis que l'obsolescence du chemin de fer s'est inversée et qu'il est devenu un mode de transport moderne alors que l'on croyait que le développement du transport serait celui du seul transport aérien. Ce retournement a modifié complètement notre regard sur les questions historiques, car l'archéologie industrielle, dans laquelle j'inclus les chemins de fer, est née dans un contexte où l'on voulait marquer un changement d'époque et un changement de culture. On pensait alors qu'il était urgent de garder les traces d'un passé promis à la disparition et dont les chemins de fer étaient l'armature, de capitaliser le souvenir, sachant que l'obsolescence était omniprésente. Le fascinant retournement, pour ainsi dire copernicien, de l'histoire du chemin de fer auquel nous avons au contraire assisté est à mon avis le résultat de deux facteurs. Le plus important me semble résider dans les effets de la conurbation; c'est le fait que le chemin de fer s'est avéré le seul moyen de transport approprié à l'existence de continuités urbaines à des échelles devenues continentales; les autres moyens de transport se sont révélés inadaptés à ce type de besoin par la pollution qu'ils entraînaient. On s'est aperçu par ailleurs que les moyens de transport ne se succédaient pas en cycles et que le mode le plus ancien ne serait pas entièrement remplacé par les suivants, le canal étant vaincu par le chemin de fer, celui-ci par la route, le transport aérien finissant par les dominer ou remplacer tous. Or les aspects les plus traditionnels du transport, comme le canal et derrière lui le chemin de fer, ont démontré une vitalité inattendue.

4 En deuxième lieu, je souhaite souligner le basculement des problématiques qui s'est produit "d'un siècle à l'autre", pour reprendre le sous-titre de notre colloque, à travers la question de l'énergie. La "crise du pétrole", si secondaire qu'elle puisse paraître avec le passage du temps, est en réalité l'indice d'une transformation culturelle très profonde de notre approche de l'industrie. Or c'est à ce moment qu'est apparu le patrimoine. On est passé de l'inventaire avant ou après décès d'un système technique considéré comme dépassé à une thématique du passage de relais. On a commencé à considérer que la culture héritée avait quelques chances d'être réinvestie dans la culture du futur, qu'elle était même un élément de compréhension de ce qui allait arriver. Alors que le patrimoine a d'abord été l'expression d'un refus de la modernité, d'une contestation du mouvement en cours et qu'il a construit son identité comme une instance d'opposition, aujourd'hui, il apparaît tout d'un coup comme un vecteur de projet. C'est ce basculement qui justifie notre présence ici. Ce n'est pas en tant que nostalgiques d'un chemin de fer condamné à devenir un décor de théâtre que nous sommes réunis, mais parce que nous réfléchissons à la fois sur le passé et sur l'avenir du chemin de fer. 
Cependant, ce sera mon troisième point, le sous-titre donné à notre débat, «Les perspectives de la recherche", me semble ambigu; mais cette ambiguïté est significative, car elle touche aux deux couples antinomiques "patrimoine et architecture, urbanisme et paysage ». "Patrimoine et architecture» ne sont pas naturellement destinés à s'entendre ni même à se soutenir réciproquement, mon expérience professionnelle à la Cité du patrimoine et de l'architecture me l'a prouvé. L'hostilité que manifestent les architectes libéraux à l'égard des architectes des bâtiments de France en est un autre exemple: ils rejettent la surveillance de leur activité au nom de valeurs qu'ils ne partagent pas. Cette antithèse est institutionnelle, elle oppose les institutions qui défendent l'image de l'héritage collectif à celles qui proclament le renouvellement ininterrompu de l'imaginaire artistique. Si donc la réunion de ces deux termes n'est pas assurée d'un résultat positif, il faut pourtant reconnaître qu'aujourd'hui, même dans les écoles d'architecture qui sont le lieu d'une défense corporatiste de l'architecture moderniste, le patrimoine fait une extraordinaire percée : il est désormais associé à la modernité d'une façon très forte et qui n'est plus contestée.

6 Quant à " urbanisme et paysage ", leur antinomie repose sur un concept aujourd'hui en passe d'être très fortement contesté, l'opposition « ville-campagne ». L'urbain, en fait, touche la totalité des espaces, y compris les espaces non urbanisés. C'est là où le regard sur le chemin de fer est novateur, parce qu'il traite non seulement des questions de paysage, mais aussi des questions d'organisation des réseaux et que, à ce titre, il fait comprendre comment des éléments dispersés entretiennent des liens très étroits. Notre problématique, dans son ambiguïé même, montre donc à quel point nos axes de pensée sont en train d'évoluer parce qu'on a compris que le paysage ouvert n'est pas plus un paysage naturel que le paysage construit des villes ; c'est un paysage habité, restructuré par l'homme, totalement transformé. L'Éden rural est une utopie qui appartient davantage au XVIII ${ }^{e}$ qu'au XXI ${ }^{e}$ siècle.

7 Après ces trois remarques préliminaires, j'en viens à l'introduction du débat, également en trois volets. Il me semble que ses thèmes traitent du paysage, des abords et de ce que j'appelle modestement le bâti, pour ne pas dire l'architecture, qui sont trois échelles différentes d'une même pratique. J'en ajouterai une quatrième en conclusion.

8 À propos du paysage, nous avons beaucoup évolué, grâce en particulier à l'action de votre association, car le regard que nous portons désormais sur les guides de voyage du $\mathrm{XIX}^{\mathrm{e}}$ siècle nous fait comprendre qu'on peut saisir la dimension de récit du paysage à travers l'observation des lignes de chemin de fer ${ }^{1}$; nous avons regardé autrement, nous avons regardé des paysages en mouvement, ce qui rejoint le thème de notre réunion. J'en trouve l'écho dans la littérature actuelle, avec par exemple Paysage Fer de François Bon, un ouvrage très proche de nous aujourd'hui ${ }^{2}$. Je pense aussi à des thèses universitaires qui ont étudié la constitution des paysages touristiques de bord de mer ou de montagne et qui montrent à quel point l'analyse des récits d'espace, en principe caractéristique de l'architecture, s'élargit aux échelles géographiques, sans limitation. Cela vient en parallèle d'autres approches, comme celles de Antoine Picon $^{3}$ ou de Claude Jasmin ${ }^{4}$ qui, en s'intéressant à la transformation de la nature par le travail de l'ingénieur, montrent l'action de l'homme, y compris dans le modelé des sols, bouleversé par l'intervention des réseaux de chemin de fer. On a donc progressivement osé parler de la mise en forme du paysage par le travail humain, ce qui est aujourd'hui une des grandes thématiques dans laquelle nous vivons. Un ouvrage comme De Paris à la 
mer en vient à écrire un trajet en chemin de fer qui fait apparaître à la fois la politique d'une entreprise investissant dans le réseau de chemin de fer et la trace définitive qu'elle inscrit dans la compréhension du paysage et dans la culture régionale ${ }^{5}$. Dans le même genre d'idées, on s'intéresse aujourd'hui à des formes d'occupation ferroviaires complexes, notamment toutes les formes un peu hybrides, comme les trains d'intérêt local empruntant la voie publique à la fin du XIX ${ }^{e}$ siècle en une espèce de partage du territoire surprenante, comme les tramways interurbains et les tram-trains qui deviennent une des modes de notre société, ou les embranchements particuliers, les voies de port... La réflexion s'intéresse à un type de chemin de fer différent de celui, mieux connu, qui est en voie propre et complètement autonome.

9 Si ces questions de paysage sont largement ouvertes, je regrette cependant l'absence de réflexions, par exemple sur les faisceaux de voies autoroutières, de chemin de fer et de transport de l'énergie à distance - les grandes lignes électriques à haute tension - qui se constituent sous nos yeux : cette manière de réunir en un seul lieu toutes sortes d'objets parce qu'ils ont la caractéristique de provoquer de la nuisance n'a pas encore fait naître une qualité de production suffisamment intéressante. Je crois que nous sommes en droit d'exiger que, dans ce domaine, une synergie créative se produise audelà de la simple association d'éléments perçus comme négatifs : c'est un axe de l'introduction des voies nouvelles dans notre paysage du siècle à venir. Il en est de même pour le développement spectaculaire de ce que j'appelle "le mobilier " par un abus de langage, c'est-à-dire, pour le chemin de fer, les fils des lignes électriques aériennes, les potences, les portiques, les signaux. Tous ces éléments se développent et occupent une place visuelle considérable dans le paysage, mais ils n'ont pas encore été traités - on le voit dans le cas des lignes à haute tension - ils ne bénéficient pas d'une démarche de design très poussée et leur qualité pourrait être grandement améliorée.

Les abords ont fait l'objet des travaux de recherche les plus importants depuis deux décennies. Ces travaux ont analysé le lien qui attache la gare au milieu urbain, lien pour lequel on peut mettre en œuvre la problématique du paysage esquissée plus haut. Les portes de ville, les gares, qui ont été la réussite de la pensée scientifique, ont été très richement étudiées. Les interventions urbaines contemporaines sont en revanche plus compliquées, moins abouties et plus contradictoires, comme Tolbiac à Paris, lieu où on a repris un modèle d'urbanisme du début $\mathrm{du} \mathrm{xx}^{\mathrm{e}}$ siècle, celui des grandes gares américaines telles Park Avenue à New York. Comme beaucoup d'opérations urbaines, ce projet créera sans doute un paysage inattendu, expression des conflits entre les acteurs.

11 Le troisième et dernier volet est le point de vue architectural. On peut resserrer encore la focale, poser la question de la corrélation des éléments à l'échelle de l'architecture. La plupart des travaux sur les grandes gares prennent aujourd'hui cette dimension en compte; ils ne traitent plus le monument comme un objet indépendant, mais comme un objet dans un contexte avec lequel il dialogue. Les opérations de rénovation menées depuis une vingtaine d'années, notamment dans le cadre du réseau à grande vitesse, exploitent cette connexion avec l'environnement urbain et architectural de la gare. Sur cette thématique de la gare s'est greffée une réflexion paradoxale sur la façon d'associer demande de monumentalité et demande de consommation : comment faire à la fois un grand supermarché et un grand monument dans un même lieu, alors que les deux projets sont en contradiction, opposant l'idée de stabilité de la représentation du point de vue du paysage au renouvellement du décor et des fonctions au quotidien? Les 
problèmes, à l'évidence complexes, qui se posent aboutissent toujours à la même question: la gare est-elle un territoire fermé pour des raisons de sécurité et de dangerosité du transport ou, au contraire, un lieu ouvert d'échange ? Le compromis est permanent. L'une des choses les plus surprenantes qui se produit sous nos yeux est en effet la revendication monumentale de la gare qui a repris sa vigueur ces dix dernières années. Les grandes gares construites pour le TGV, dans leur recherche du prestige, sont bien des monuments du $\mathrm{xx}^{\mathrm{e}}$ siècle. En revanche, je serai plus prudent dans mon appréciation d'autres aspects de l'étude architecturale du bâti. L'analyse des types architecturaux a en effet tendance à s'enfermer dans une réflexion un peu étroite sur l'architecture réglementaire normalisée, en faisant entrer les gares dans le système qu'on connaît pour les casernes, les écoles, etc. La description de la rationalisation des types n'est pas à mon avis la seule réponse à apporter sur l'architecture de la gare. Par ailleurs, la gare n'est pas tout. Quantité d'autres constructions extrêmement intéressantes sont dignes d'être analysées, éventuellement d'être conservées. Je prendrai deux exemples qui ont fait l'objet de débats récents: ce sont la gare des Batignolles, dont le destin sera la disparition pure et simple, parce que c'est un grand objet qui prend beaucoup de place et dérange toutes les politiques d'aménagement, et la halle du Sernam d'Austerlitz, dérangeante aussi, mais que quelques acteurs sont décidés à prendre en compte.

Il est évident que, voici quinze ans, tous ces bâtiments auraient été rasés dans un silence total alors qu'aujourd'hui des doutes, des hésitations, des conflits se font jour. Le patrimoine commence toujours avec la contestation, il peut ensuite progresser.

Je souhaite ajouter une quatrième échelle à l'analyse, qui n'est pas architecturale, ou du moins c'est une architecture non située : tout ce qui est design, tout ce qui est objet et que j'appelle "une architecture sans site». Je pense à l'architecture intérieure des voitures, à celle des bâtiments fonctionnels, à celle des équipements techniques, une architecture dont la durée est moins longue, tout ce travail du design ferroviaire qui a des proximités avec notamment le monde de la mode. Je pense que la réflexion pourrait être approfondie, car nous disposons d'une richesse de documentation, d'information, de signification aussi de cette production qui mériterait d'être valorisée. Je prends un exemple: il existe toute une réflexion sur le bruit du roulement, sur le silence et le confort acoustique à l'intérieur des voitures qui me semble assez peu prise en compte par nos réflexions. Si l'on pouvait comparer des enregistrements concernant 150 ans de matériel ferroviaire, on constaterait que l'environnement sonore est un monde qui a complètement changé, sans doute sous l'influence de l'aviation ou de l'automobile. Nous n'avons pas entendu, précisément, combien il y avait là une création artistique.

\section{Évelyne Lohr}

Je remercie Karen Bowie de m'avoir invitée à participer à cet échange sur les perspectives de la recherche. Je travaille au sein d'un conseil général, celui de la SeineSaint-Denis, territoire urbain tourmenté et très marqué, comme beaucoup d'autres territoires de la banlieue, à la fois dans l'histoire de sa constitution et aujourd'hui dans ses paysages, par les infrastructures de transport, notamment ferroviaires. C'est un territoire concerné actuellement par de très importantes mutations, dans lesquelles, là encore, les emprises ferroviaires ne sont pas en reste. On a assisté dans les dernières années à la modernisation du faisceau nord, à l'arrivée du RER B, du TGV Nord, puis, sur le faisceau est, du RER E, du TGV Est avec l'installation de nouveaux équipements de maintenance très importants; parallèlement, on constate le déclin de la desserte fret 
en milieu urbain dense et des embranchements industriels qui suivent la désindustrialisation de la banlieue, la fin des grands triages urbains, le déclin des grandes gares de marchandises et des études pour l'installation de plates-formes à caractère multimodal plus restreintes. Enfin, dans le domaine du transport de voyageurs, c'est la mise en route d'un certain nombre de nouvelles dessertes ou la reprise d'anciennes dessertes, comme le tram-train entre Bondy et Aulnay ou le projet de ligne «tangentielle» sur le tracé de la Grande Ceinture. Dans ce mouvement qui affecte le territoire coexistent la modernisation et la désaffectation ou l'obsolescence d'un certain nombre de lignes et d'équipements. Notre Service du patrimoine culturel tente de contribuer à la compréhension de ce territoire complexe et peu aimé et de lui apporter une cohérence, à travers son analyse rationnelle et documentée, et essaie de forger des outils pour contribuer à une évolution plus soucieuse de l'existant qu'elle ne l'est. Nous nous plaçons bien dans cette perspective d'un patrimoine qui est un moteur d'évolution et non un objet de nostalgie.

Le point de départ de notre démarche est la connaissance du territoire, donc son inventaire, qui peut être communal, territorial, cantonal, thématique. S'intéresser au chemin de fer n'est pas une chose aisée, puisque le réseau de ce patrimoine linéaire se moque des limites administratives; qui plus est, le chercheur entre dans un univers technique extrêmement difficile d'accès pour qui ne le connaît pas intimement; enfin, il est difficile de plaider pour sa sauvegarde car, surtout dans ce territoire de banlieue si ingrat du point de vue de l'occupation du territoire, il représente d'énormes emprises, des extra-territoires très étendus et causes de nuisance dont le patrimoine technique est difficile à défendre.

Figure 1. Copie d'écran de la page Internet : http://www.atlas-patrimoine93.fr/documents/cartei/ index.html

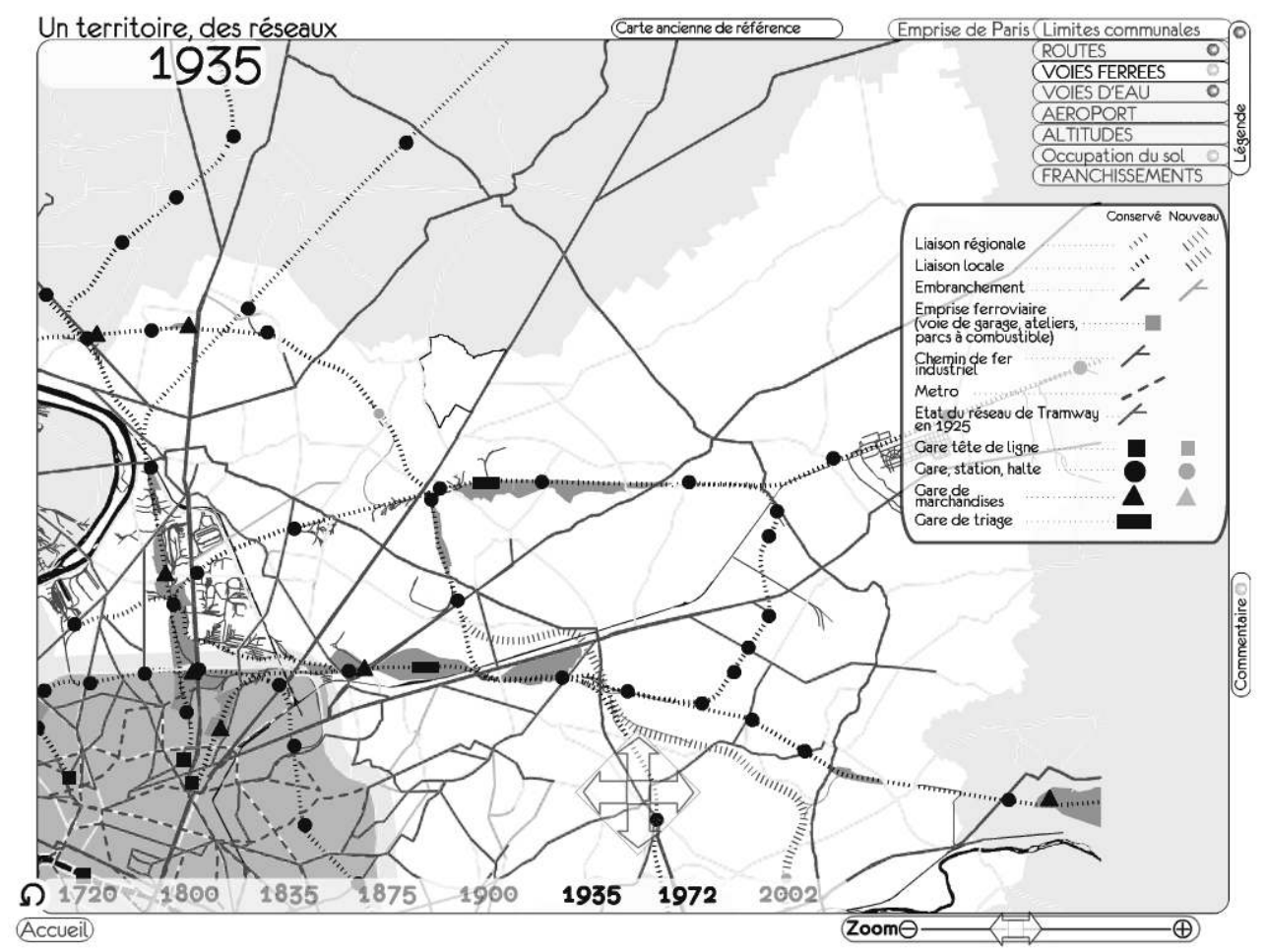



l'évolution des infrastructures de transport, celle de la construction de notre territoire depuis le début du xviIIE siècle jusqu'à nos jours. Dans ce document de 1935 (fig. 1), on voit l'ensemble des infrastructures situées à l'arrière de la gare du Nord et de la gare de l'Est, gare de marchandises, gare de triage et chemin de fer industriel qui occupent un espace très important dans la plaine Saint-Denis et sur les communes de Pantin, Bobigny et Le Bourget. Au-delà, le faisceau se réduit en largeur et l'emprise redevient linéaire. Les territoires se sont mobilisés pour obtenir des dessertes locales, d'où une urbanisation «en tache d'huile» qui a pour résultat un tissu pavillonnaire lié au chemin de fer, dont les habitants ont été soumis à des mouvements pendulaires de plus en plus lointains. désormais bien étudié (fig. 2). Après l'installation des têtes de lignes à l'intérieur des fortifications dans ce qui allait bientôt être Paris, l'augmentation croissante du trafic a généré des équipements à l'arrière, toujours plus loin, franchissant les anciennes fortifications pour s'étendre dans de grands espaces plus faciles à exproprier. La gare de La Chapelle franchit la limite de Paris pour s'installer sur le territoire de Saint-Denis. L'extension du chemin de fer au cours du xix siècle vient contrarier un ordre paysager préexistant; dans le même temps il contribue à valoriser les espaces voisins, il génère une urbanisation dont la densité va à son tour contraindre la gare dans son développement. Cela explique une géographie peu rationnelle, extrêmement complexe, avec une gare qui a été obligée de s'agrandir vers la périphérie, en faisant se succéder les gares de marchandises de La Chapelle, La Chapelle-triage, La Chapelle-charbons, puis la gare de marchandises de La Plaine-Saint-Denis, jusqu'à la gare du Bourget située plus loin vers le Nord, gare de triage qui assure la régulation d'un trafic très important.

L'inventaire s'intéresse aussi à l'intérieur de cet extra-territoire où l'on trouve un certain nombre d'immenses bâtiments dont la réaffectation est très difficile et s'inscrit dans une problématique très proche de celle du patrimoine industriel en général.

Il en est ainsi des immenses halles de transbordement de la gare de marchandises de Pantin, datant des années 1860-1870, époque de l'origine de la gare de marchandises à Pantin (fig. 3). 
Figure 3. Les halles à marchandises de Pantin, avril 2006.

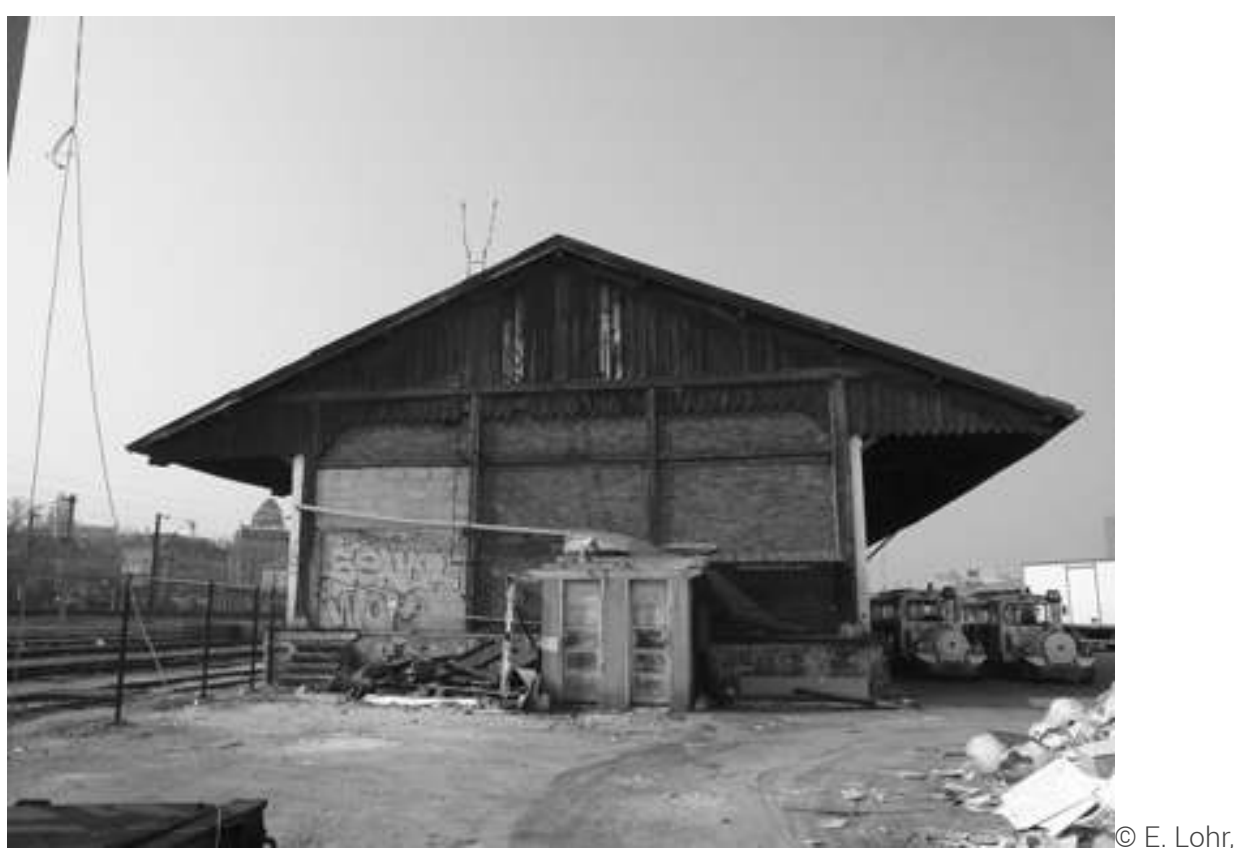

Département de la Seine-Saint-Denis

20 À Pantin se trouve également un bâtiment d'intérêt patrimonial beaucoup plus récent, dû à l'ingénieur Bernard Lafaille (1900-1955). Il s'agit d'une halle pour le trafic accéléré des marchandises, elle aussi désaffectée, qui sera amenée à se transformer en profondeur ou à disparaître, du moins en partie (fig. 4).

Figure 4 (a et b). Les halles à trafic accéléré de Pantin, Bernard Lafaille, ingénieur, 1946-1947.

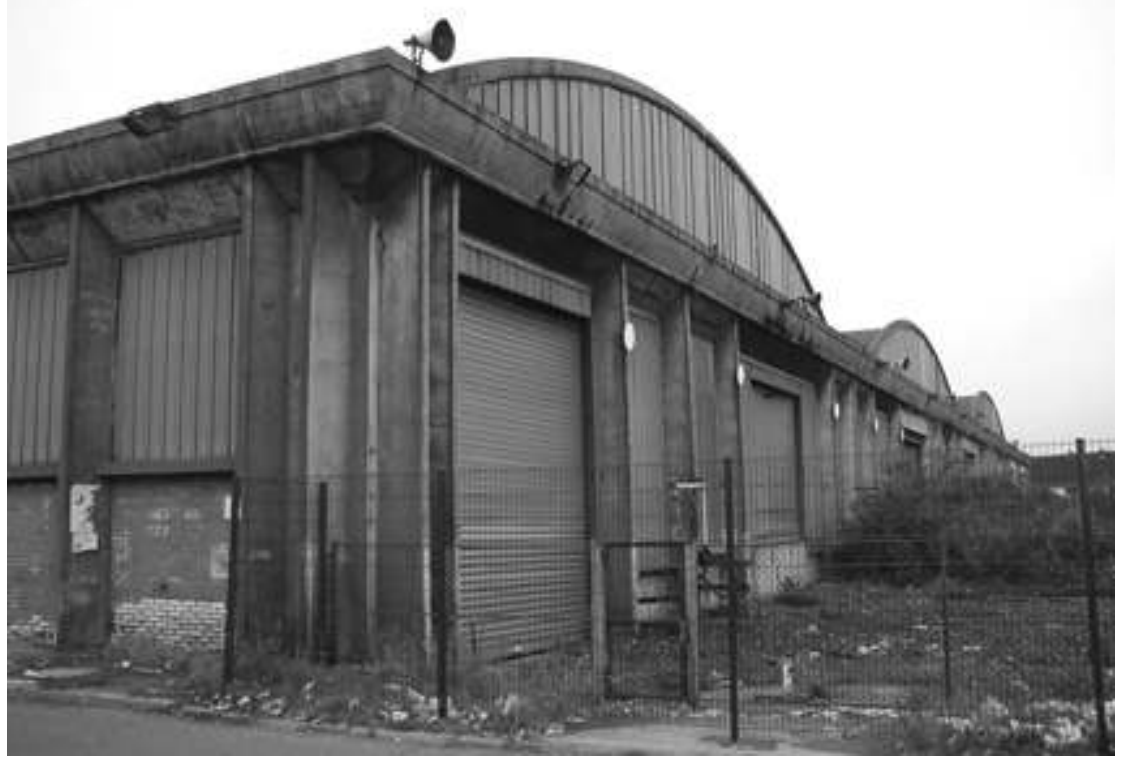






(c) Antoine Furio, Département de la Seine-Saint-Denis, 2005.

Le dépôt de La Plaine, un atelier d'entretien et de réparation des locomotives, a fait l'objet d'une protection au titre des monuments historiques temporaire de trois ans le 23 mars 2004, dans l'idée de trouver dans ce laps de temps la réaffectation indispensable à sa survie. On disait alors de ce type de protection (qui ne connait aucun fondement juridique, une protection ne pouvant être limitée dans le temps) qu'elle ne génèrerait pas de périmètre de protection autour de l'élément protégé, ce qui a permis, malheureusement, le démontage de la plaque et du faisceau rayonnant qui étaient à proximité immédiate du dépôt. Or un bâtiment n'est pas isolé, il est lié à un environnement technique. Aujourd'hui la plaque tournante ne se trouve plus sur le site, même si on peut noter qu'elle a été récupérée par une association et remontée dans un autre lieu (fig. 5). 
Figure 5 ( $a$ et b). Le dépôt des machines de La Plaine, l'atelier de levage (a) et le petit entretien (b), 1945-1952.
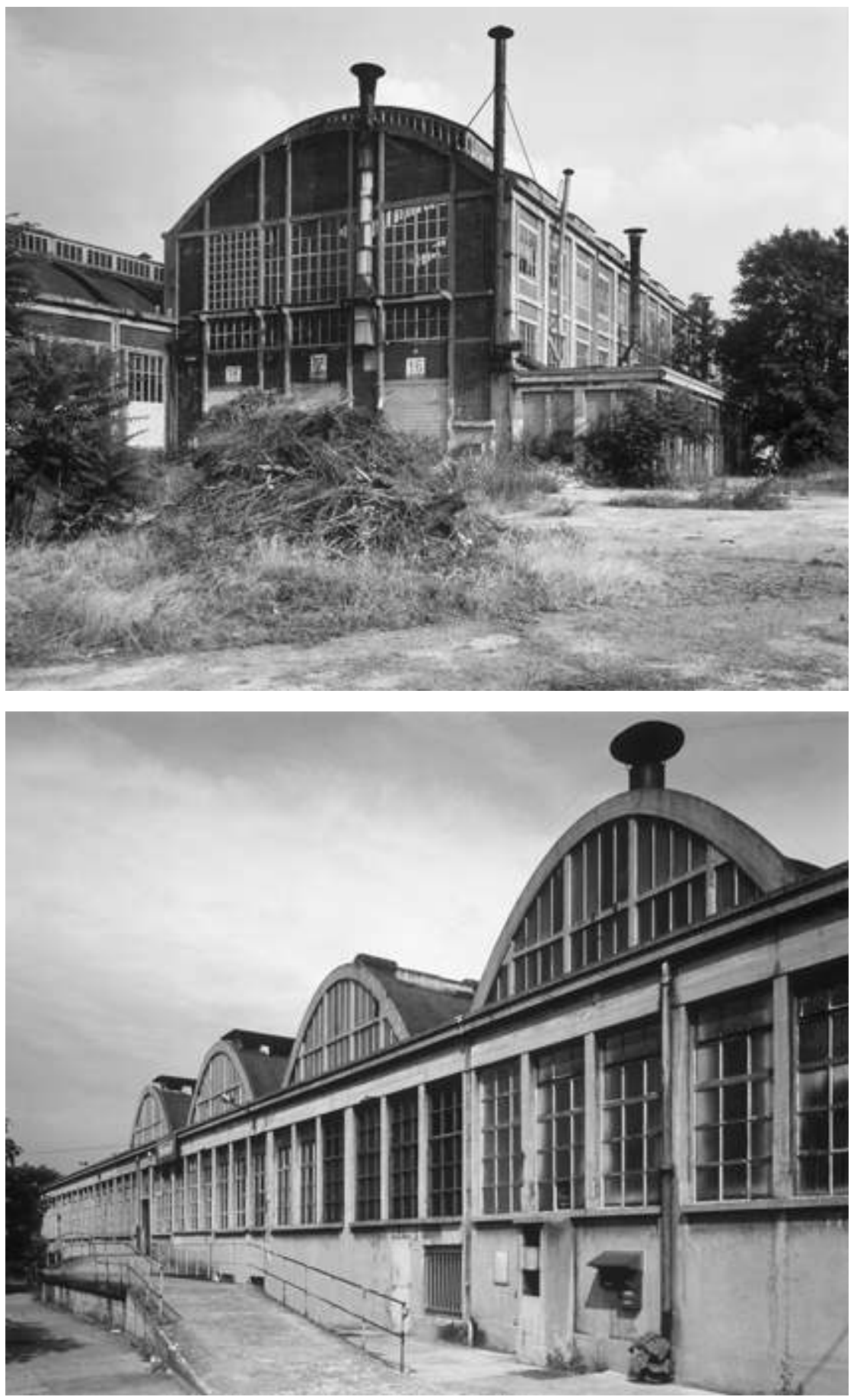

(c) L. Desmoulins, Département de la Seine-Saint-Denis

22 L'histoire des docks de Saint-Ouen illustre les impacts des réseaux sur l'évolution du territoire (fig. 6). Comme La Plaine-Saint-Denis, qui étaient structurée par un réseau de chemin de fer industriel d'initiative privée (fig. 7), les docks de Saint-Ouen, vaste zone d'entrepôts reliée à la Seine et au chemin de fer du Nord, étaient parcourus par un réseau d'embranchements ferrés. Quand on s'intéresse à la structuration de l'espace dans ce territoire en mutation, on aimerait conserver une partie de cette infrastructure 
technique pour comprendre comment elle a entièrement modelé l'espace industriel et celui des entrepôts à La Plaine.

Figure 6. Docks de Saint-Ouen, extrait de l'Atlas du Département de la Seine, révisé en 1936, feuille de Saint-Ouen.

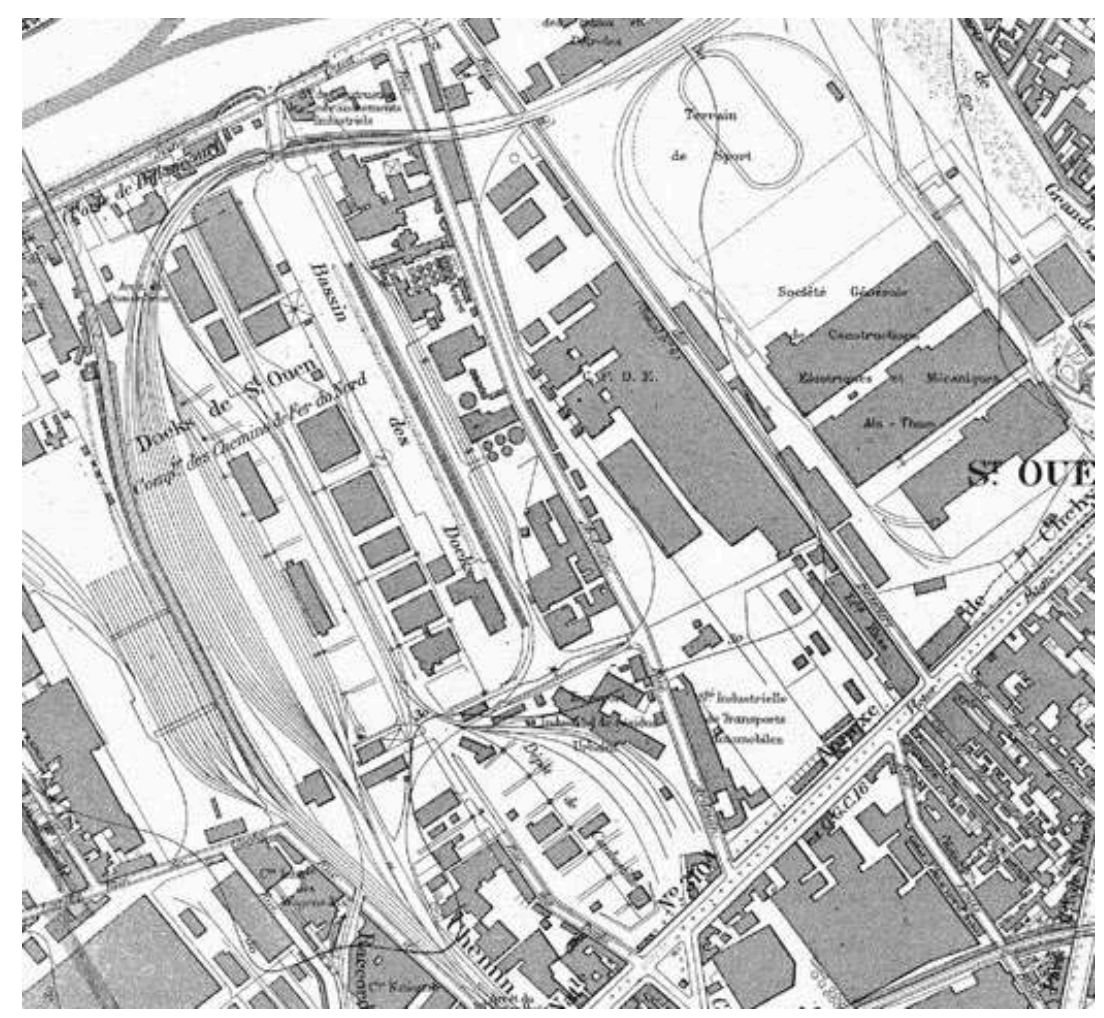


Figure 7. Plan du Chemin de fer industriel de La Plaine-Saint-Denis et d'Aubervilliers (1883 ?)

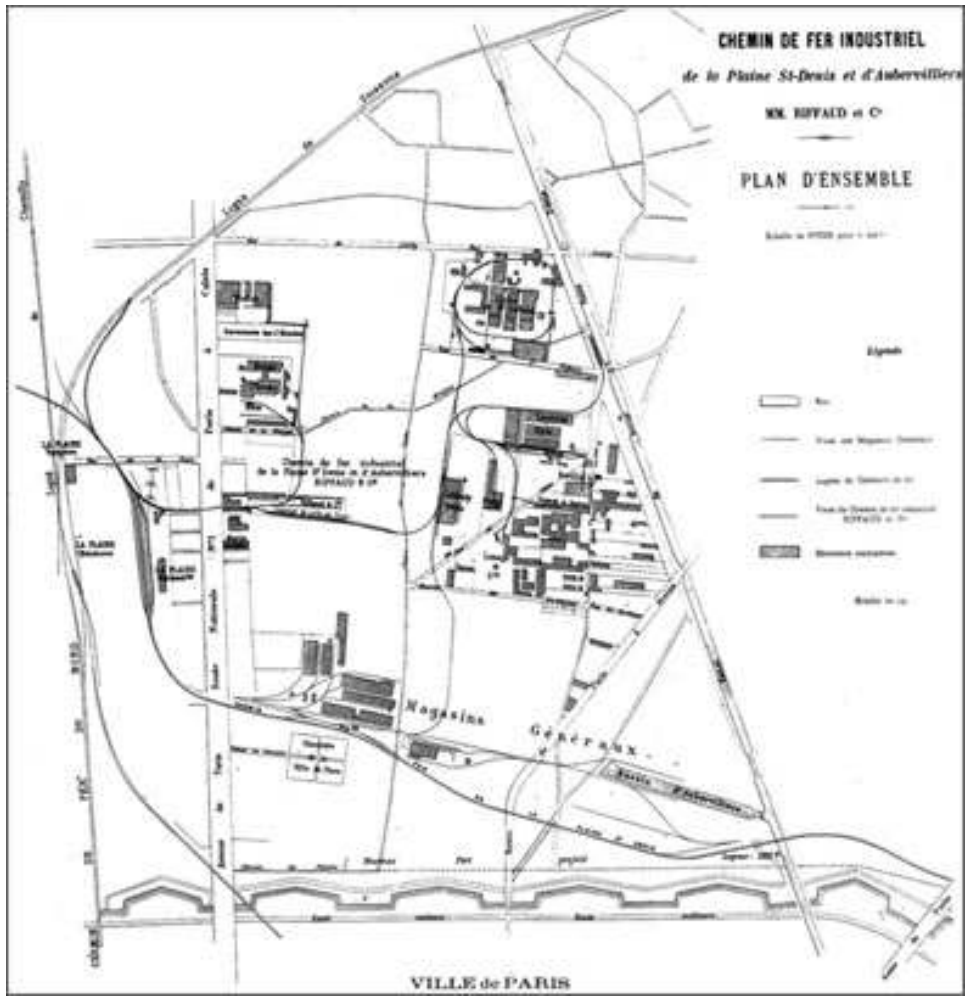

Archives des EMGP, Archives de Paris. d'intérêt local construite en 1875 dans le cadre de la loi de 1865 sur les lignes d'intérêt local à l'initiative d'un industriel, Gargan, fabricant de wagons de chemin de fer. Elle était pensée comme une ligne de desserte pour l'industrie entre ces deux communes, reliant le réseau Est au niveau de Bondy et le réseau Nord au niveau d'Aulnay. L'effet de cette ligne de chemin de fer ne fut pas tant l'industrialisation que la constitution d'un tissu pavillonnaire dans la forêt de Bondy: la ligne de chemin de fer est en effet installée sur une ancienne allée de la forêt et a la particularité, au lieu de montrer l'arrière de la ville depuis le train, d'avoir donné naissance à une ville qui se tourne vers le chemin de fer.

Lors de la réaffectation de cette ligne devenue un tram-train, la mobilisation locale d'associations et d'habitants a permis la sauvegarde de deux gares : la gare de l'Abbaye, la plus ancienne, qui date de la construction de la ligne en 1875, s'apparente davantage à une maison de garde-barrière (fig. 8a); la gare de Raincy-Pavillons témoigne de l'extension et de la modernisation du réseau au début $\mathrm{du} \mathrm{xx}^{\mathrm{e}}$ siècle. Plus grande, elle rejoint la typologie du réseau Est (fig. 8b). En revanche, la gare de l'Allée de la TourRendez-vous fait partie des bâtiments détruits (fig. 8c). 
Figure 8a. La gare de l'Abbaye, sur la ligne de tram-train de Bondy à Aulnay, carte postale ancienne.

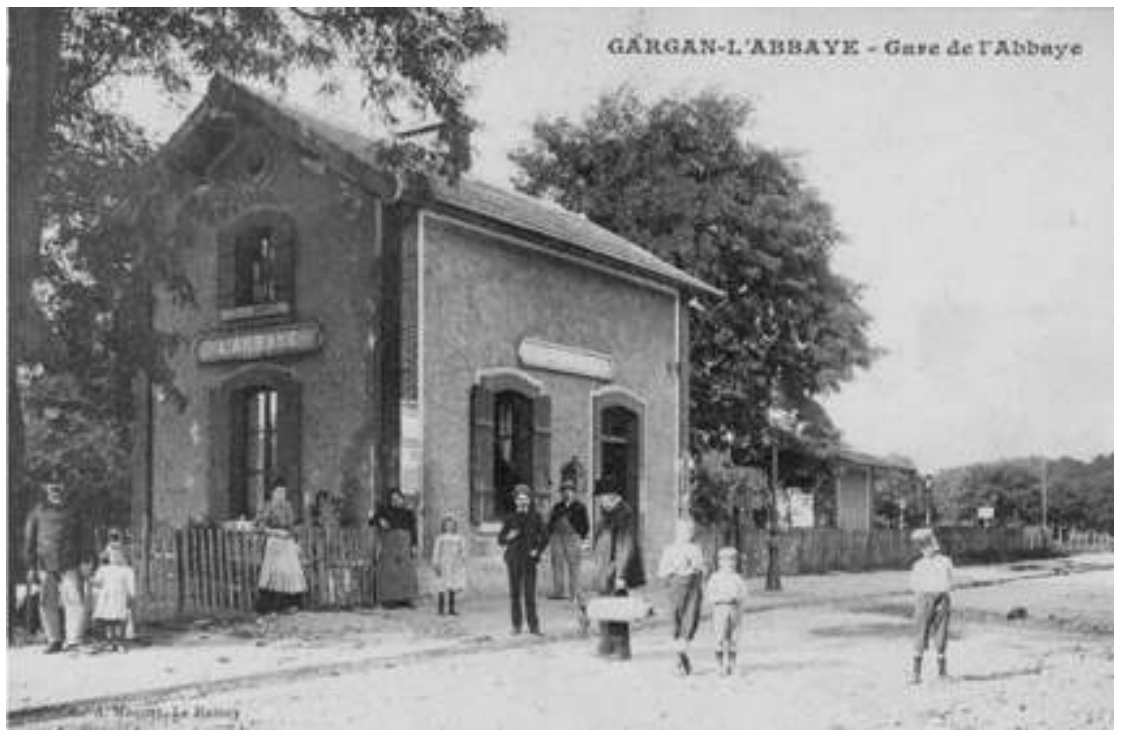

(ㄷ) Archives départementales de la Seine-Saint-Denis.

Figure 8b. Plan et élévations du bâtiment voyageurs de Raincy-Pavillons, Archives SNCF.

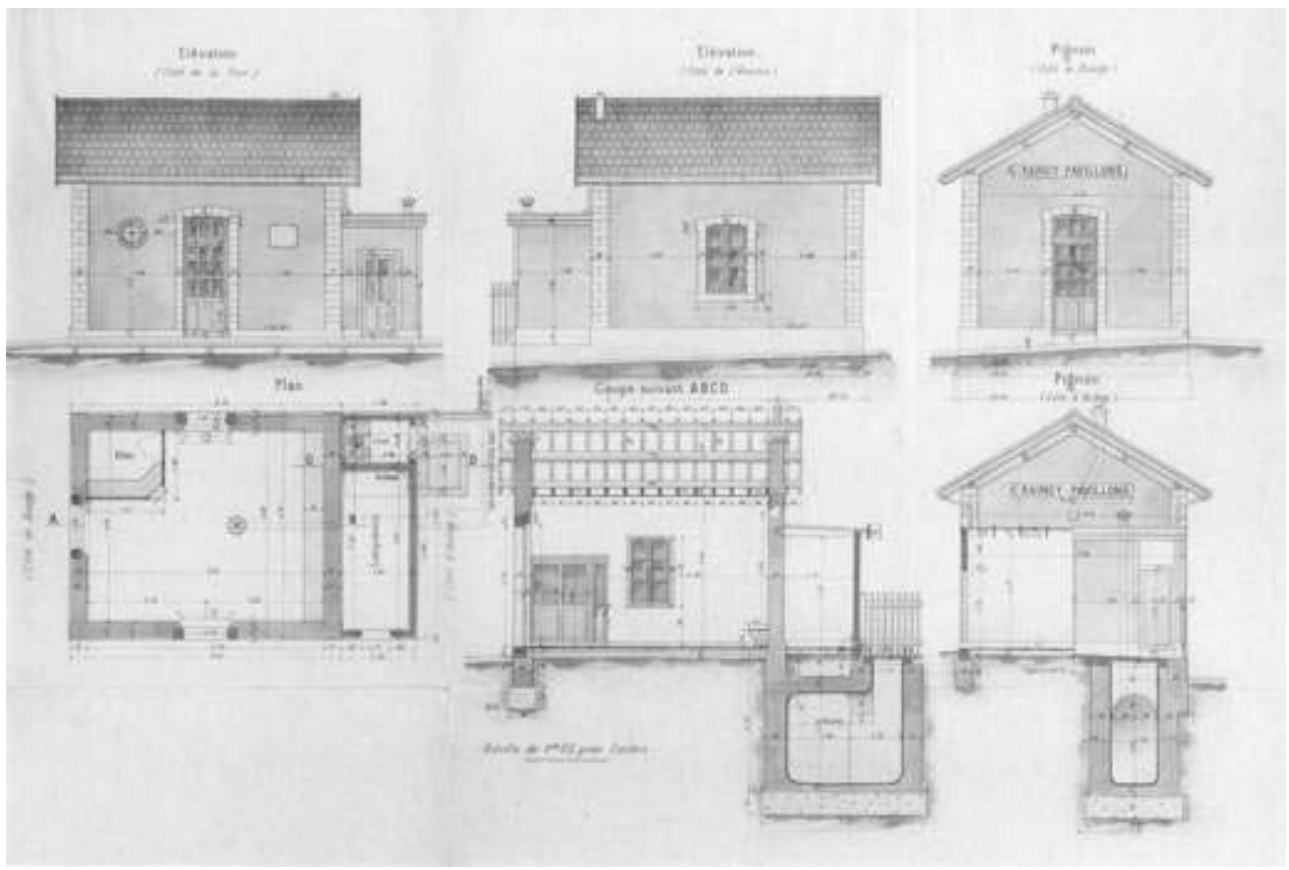


Figure 8c. La gare de l'Allée de la Tour-Rendez-vous, sur la ligne des Coquetiers, aujourd'hui ligne du tram-train Bondy-Aulnay. Bâtiment voyageurs construit entre 1908 et 1914 et détruit en juillet 2006

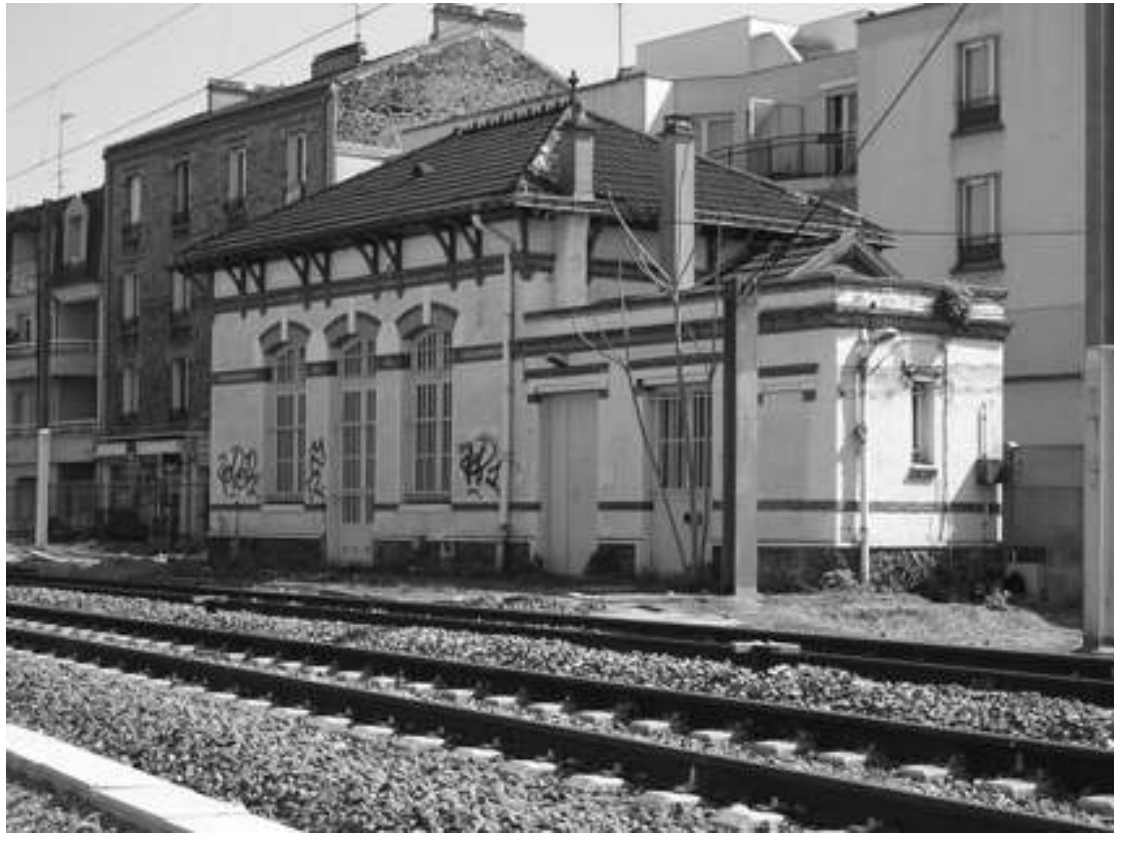

(c) A. Furio, Département de la Seine-Saint-Denis, 2005.

25 Le chemin de fer a été facteur d'urbanisation. Ainsi les lotissements d'Aulnay-sous-Bois ont été promus par des affiches de la Compagnie du Nord qui vantait leur desserte à 15 minutes de Paris par 80 trains chaque jour. La promotion des nouveaux territoires de banlieue passe par le chemin de fer (fig. 9).

Figure 9 ( $a$ et $b)$. Paysage pavillonnaire le long de la ligne des Coquetiers.

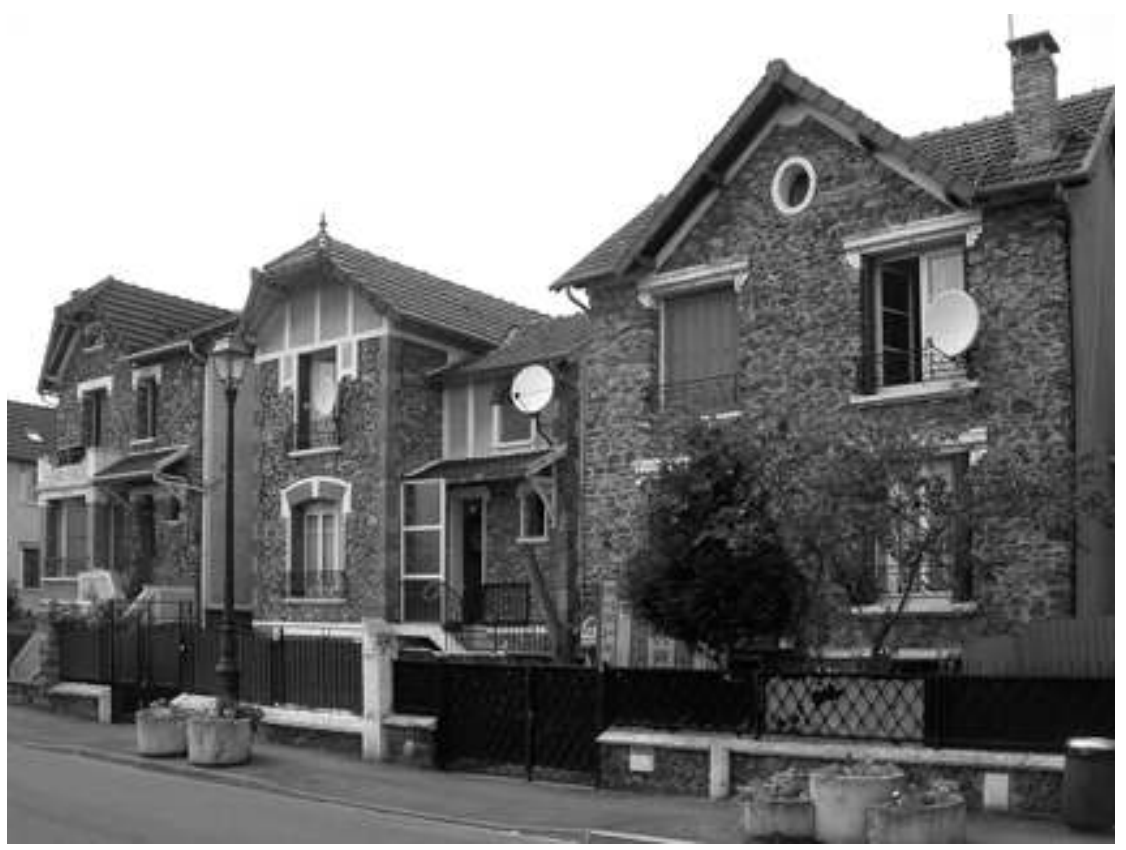




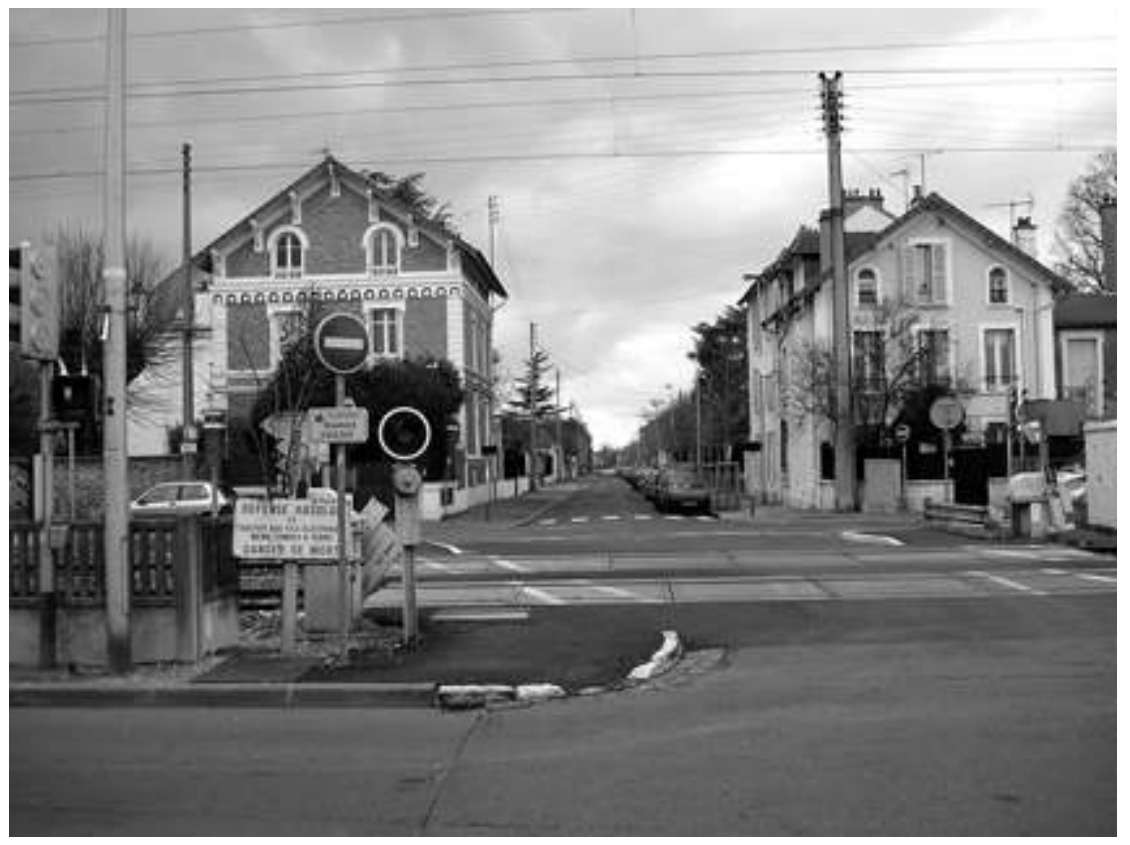

(C) E. Lohr, Département de la Seine-Saint-Denis.

Cependant, pour mettre un frein à la prolifération chaotique de la banlieue pavillonnaire, les édiles ont tenté entre les deux guerres d'utiliser le chemin de fer pour promouvoir un développement plus rationnel et contrôlé du territoire qui s'inscrit dans le mouvement des cités-jardins. La cité-jardin d'Orgemont, à cheval sur les communes d'Épinay et d'Argenteuil, est ainsi reliée à Paris par le chemin de fer (fig. 10a). Pour La Courneuve et Stains, Auburtin dessine en 1924 un projet d'urbanisation du territoire qui inclut une gare et propose une desserte par train. Il ne sera pas réalisé (fig. 10b, c, d). 
Figure 10a. Publicité montrant la localisation de la cité d'Orgemont

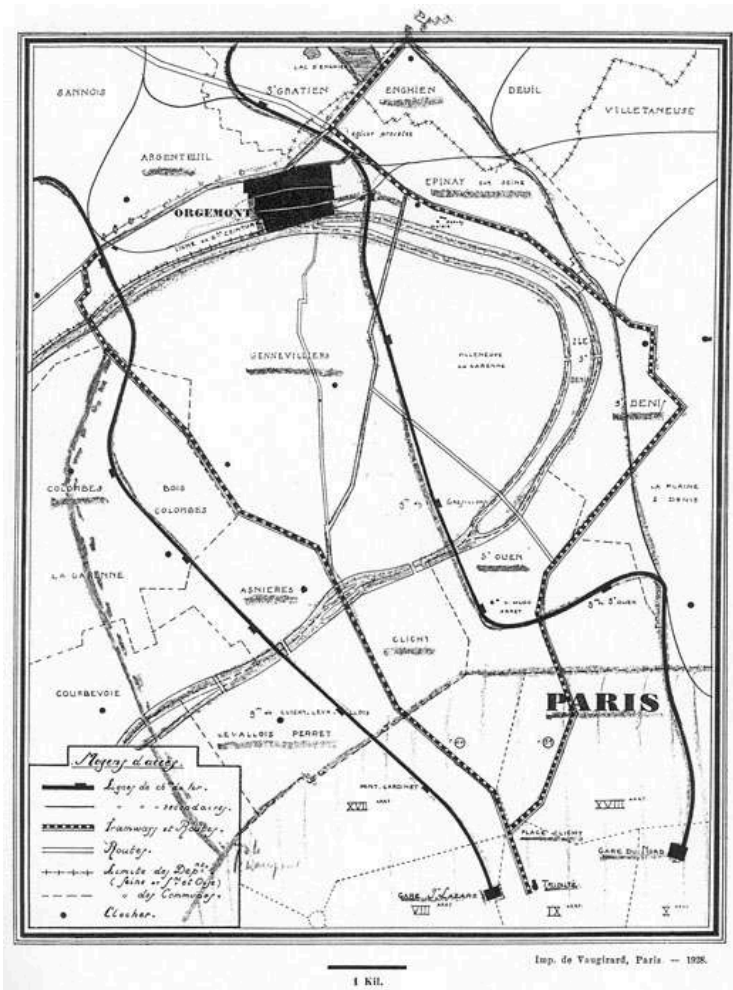

(c) Archives de la Société des cités-jardins de la Région parisienne. 
Figure 10b. Les projets de Marcel Auburtin (1872-1926) pour Orgemont, La Courneuve et Stains, extrait de : Marcel Auburtin, L'Urbanisme d'une région parisienne. Avant-projet d'aménagement des terrains situés sur les communes de La Courneuve, Le Bourget, Dugny et Stains, mémoire «Transports », Paris, 1924, 42 pages et annexes, collection privée.

PLANCHE VIII

VOIES DE COMMUNICATION PRINCIPALES



Figures $10 \mathrm{c}$ et $10 \mathrm{~d}$. Projet non réalisé d'Auburtin pour La Courneuve et Stains, ibid., collection privée.

CARTE GÉNÉRALE DES CHEMINS DE FER

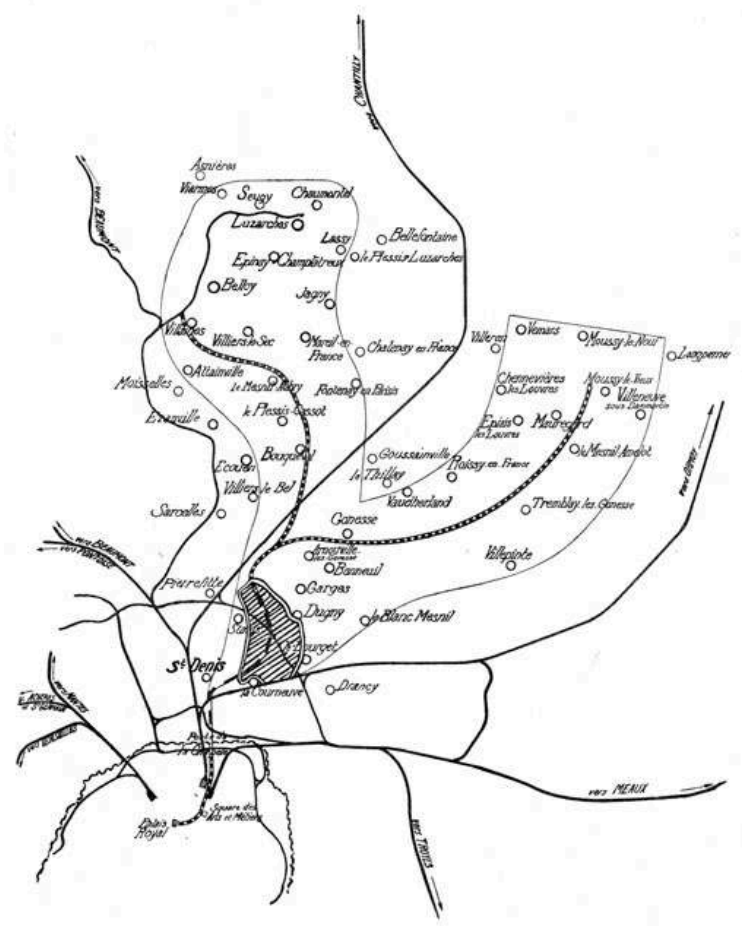






27 Le chemin de fer est créateur d'un paysage qui lui est propre. Au-delà des équipements techniques et industriels qui se trouvent dans son emprise, le chemin de fer a généré un certain nombre de bâtiments dans la ville. On pense au logement cheminot, mais ce sont aussi des équipements à caractère social et sanitaire qui s'adressent au monde cheminot (fig. 11).

Figure 11 ( $a$ et $b$ ). Cité cheminote, ensemble de logements HBM, Urbain Cassan, architecteingénieur, 1929-1930.

Figure 11 a.

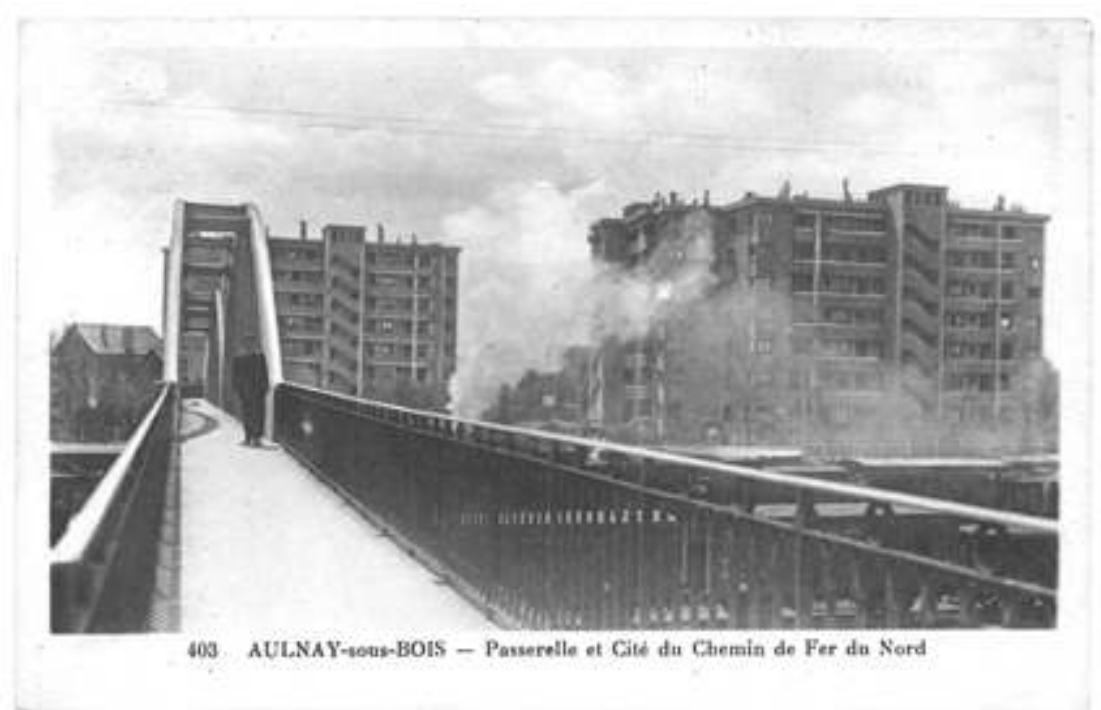

Carte postale ancienne. 
Figure $11 \mathrm{~b}$.

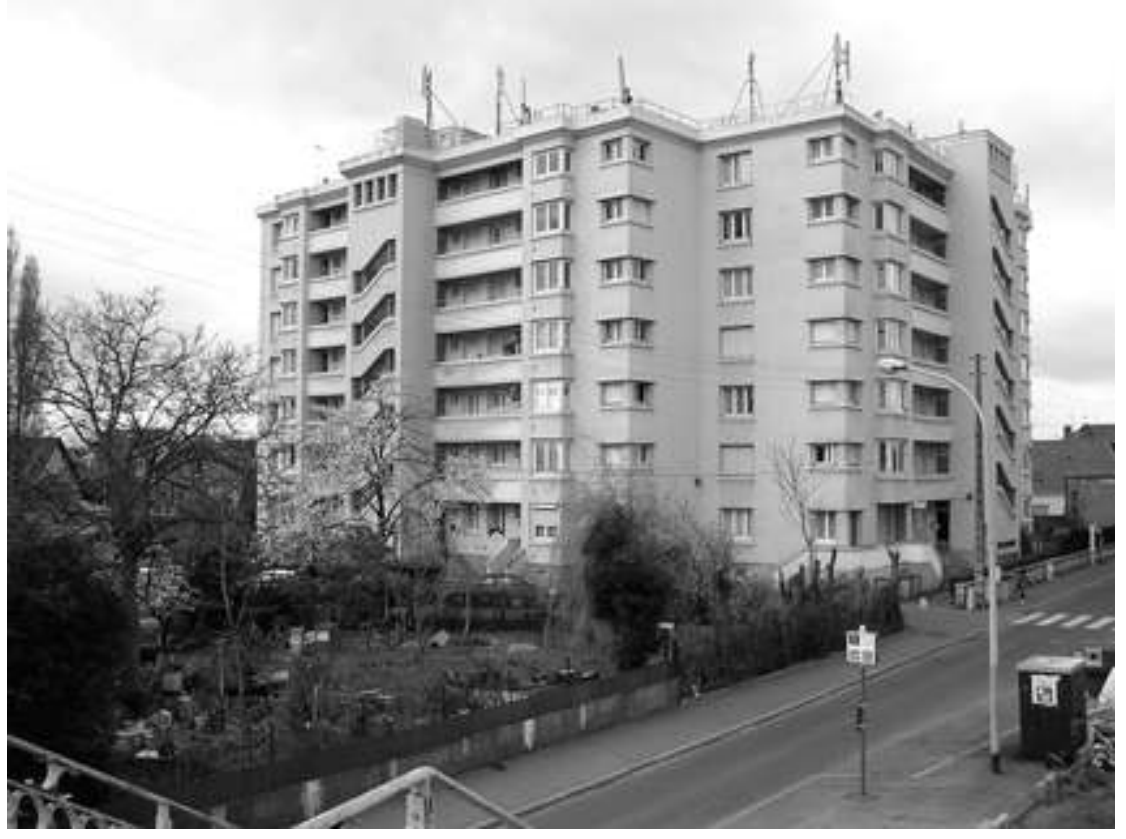

(c) E. Lohr, Département de la Seine-Saint-Denis (b).

Figure 11c. Pavillon cheminot à Aulnay-sous-Bois, Compagnie du chemin de fer du Nord, 1911.

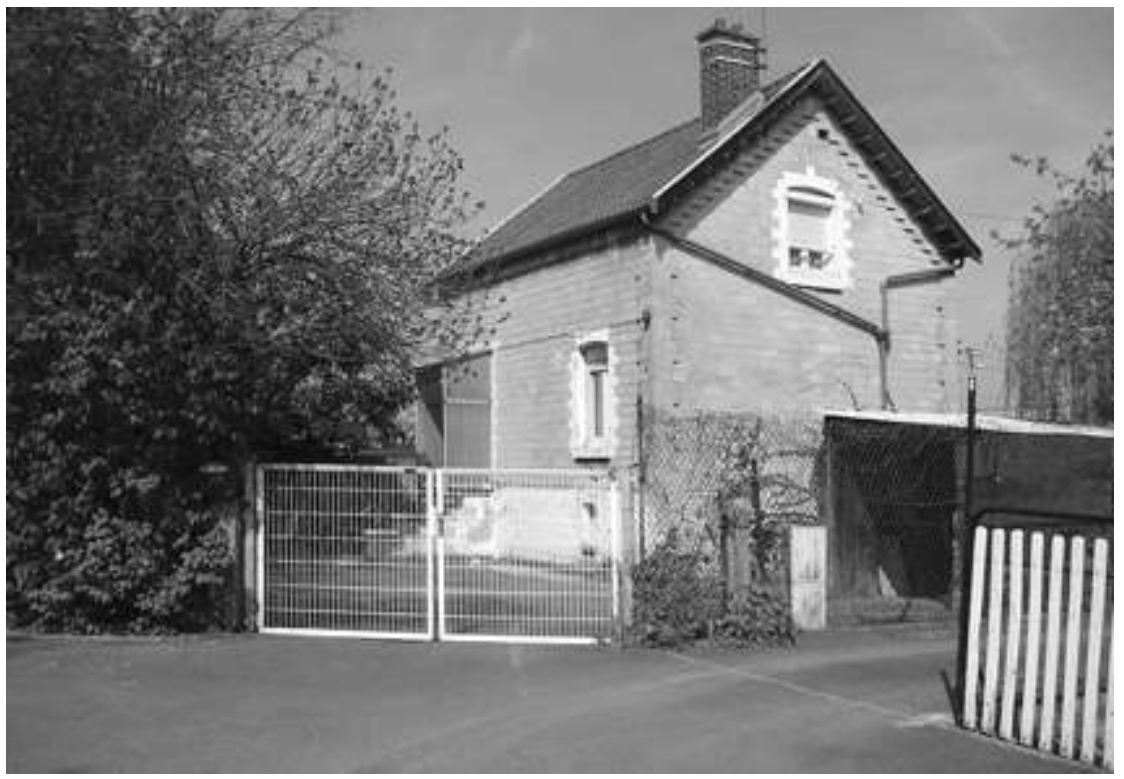

(c) E. Lohr, Département de la Seine-Saint-Denis.

Figure 11d. Cité-jardin Paul-Bert à Drancy ; Joseph Bassompierre et Paul de Rutté, architectes, OPHBM de la Seine, 1920-1922. 


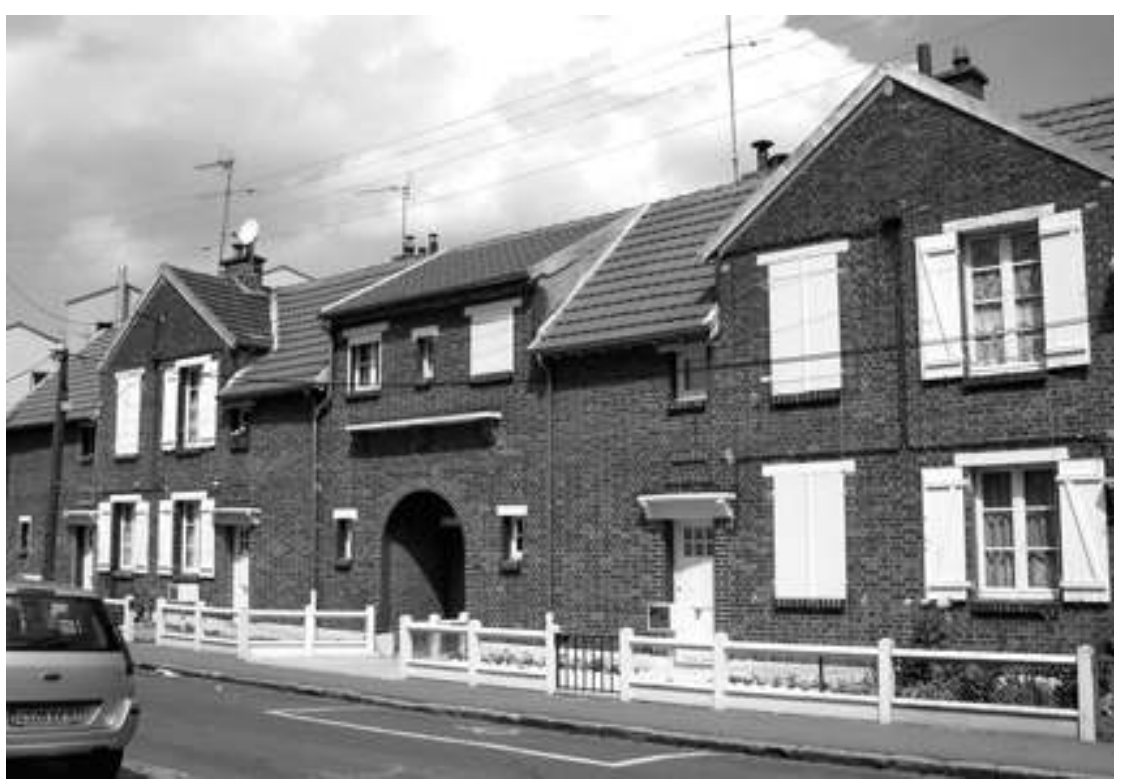

(c) B. Pouvreau, Département de la Seine-Saint-Denis.

Un exemple d'action d'inventaire conduite par le Service du patrimoine de la SeineSaint-Denis concerne la tangentielle Nord qui reprend le tracé de la Grande Ceinture, ligne jusqu'ici dédiée au trafic fret et qui va être réouverte au trafic voyageurs (fig. 12). Devant l'importante reconstruction qu'implique ce projet nous tentons de promouvoir un inventaire du patrimoine technique, au même titre que l'inventaire des bâtiments ferroviaires, notamment des gares, qui est désormais usuel et possède ses méthodes, en particulier l'étude des typologies et des modèles. Avec la collaboration de l'AHICF et du Centre d'étude d'histoire des techniques et de l'environnement (CNAM), nous nous sommes donc lancés dans un inventaire technique qui présente de grandes difficultés, la moindre n'étant pas l'accès aux installations. Il s'agit, comme dans le cas des gares, d'un patrimoine de réseaux. Cette étude de l'infrastructure technique et fonctionnelle prend en compte à la fois l'étude les ouvrages d'art - souterrains, ponts-rail, pontsroute, viaducs, murs de soutènement - mais aussi l'ensemble des voies: les rails, traverses, fixations, le ballast, ainsi que l'alimentation électrique, les supports de caténaires, les caténaires et sous-stations, et la signalisation. Notre analyse comporte bien entendu l'identification et la description de l'élément, mais surtout met en évidence son appartenance à un sous-ensemble technique, à un assemblage ou à une série. 
Figure 12a. Estacade de la ligne complémentaire de la Grande Ceinture à Neuilly-sur-Marne entre le boulevard du Maréchal Foch et la Marne.

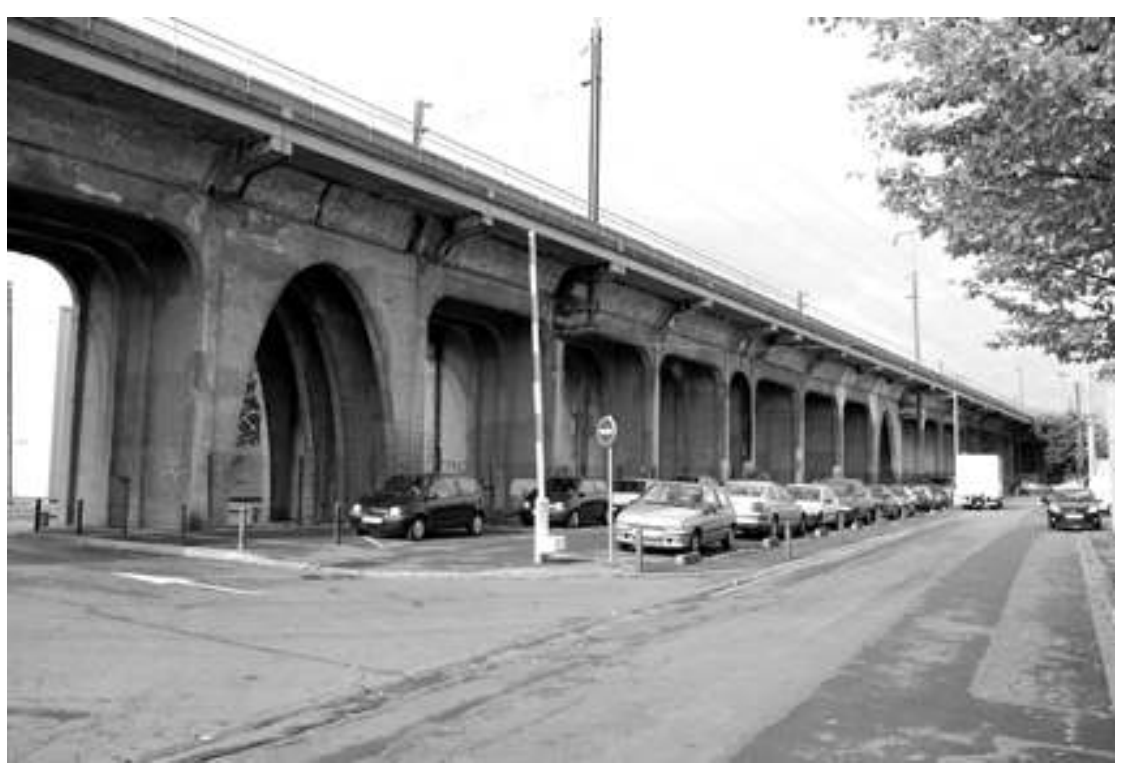

(C) Gérard Jigaudon, CNAM / Département de la Seine-Saint-Denis

Figure $12 b$, le poste d'aiguillage du triangle de Gagny, Grande Ceinture.

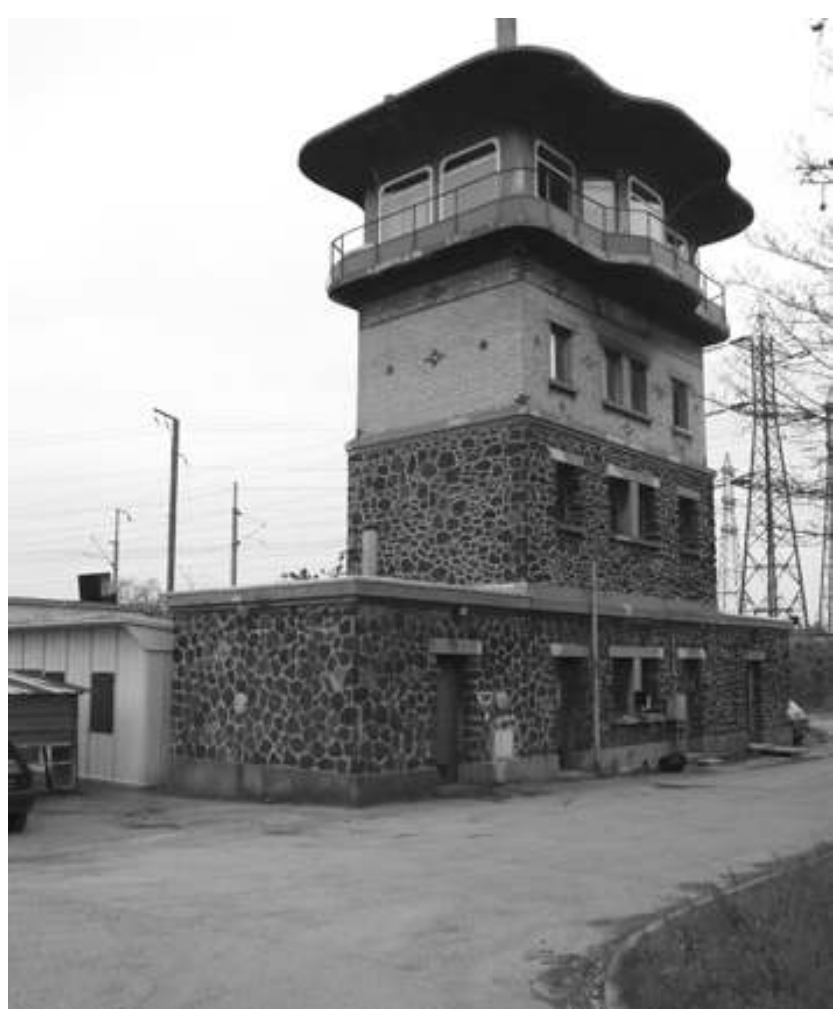

(c) Guillaume Tozer, Département de la Seine-Saint-Denis

29 Un autre volet de nos interrogations concerne le paysage ferroviaire. Les grands espaces ouverts au sein des territoires denses de banlieue, comme la gare de triage de Drancy, sont amenés, à terme, à être pour le moins restreints, sinon effacés dans le cadre de ce qu'on appelle renouvellement, « re-couture urbaine », « renouvellement de 
la ville sur elle-même »... Les villes sont en effet impatientes de récupérer ces grands espaces pour y reconstituer un tissu urbain et créer un lien entre des quartiers qui ne sont reliés que par des passerelles longues de plus d'une centaine de mètres. Pourtant, nous souhaitons promouvoir l'idée d'un inventaire des paysages, des points de vue... Quand le chemin de fer traverse ce territoire, s'il est parfois à niveau, il est très souvent en talus. Depuis le train, depuis les ponts, la vue plonge dans ces territoires ferroviaires pourtant fermés. Un inventaire des points de vue permettrait de réfléchir sur le potentiel des paysages ferroviaires dans la perspective de l'évolution en cours (fig. 13).

Figure 13a. Le triage du Bourget, s.d.

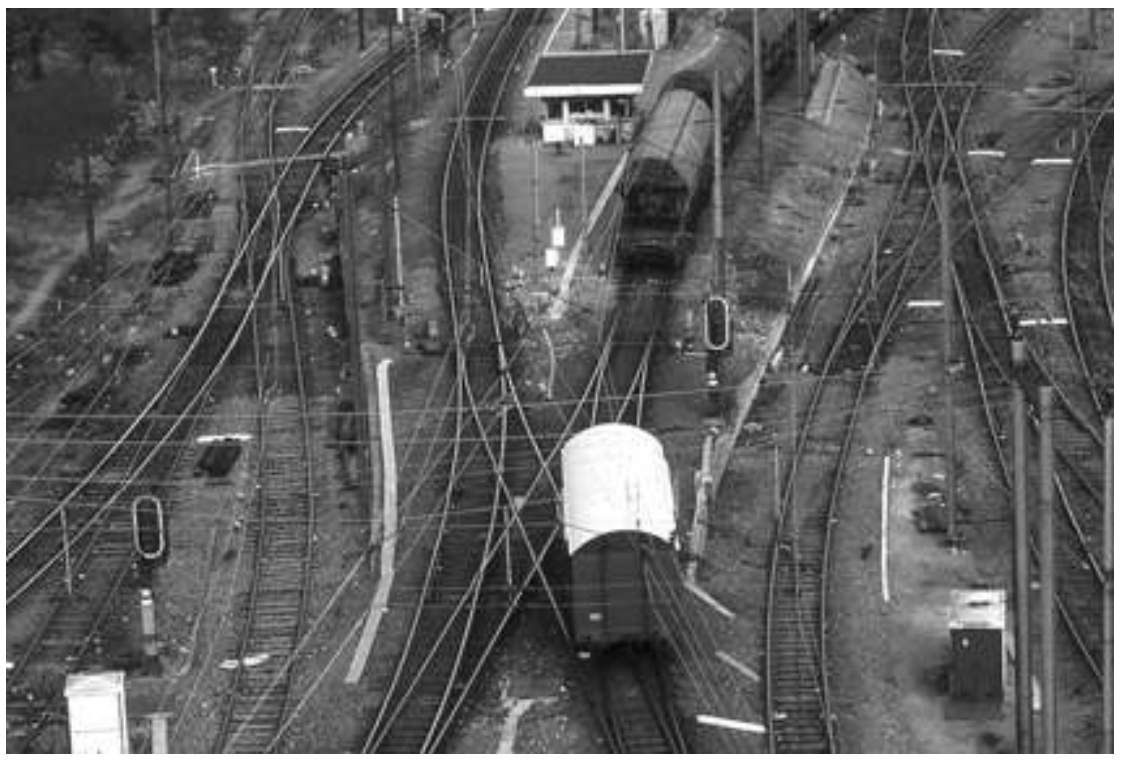

(c) SNCF / CAV.

Figure 13b : Noisy-le-Sec - Passage des voies impaires Paris-Meaux sous la Grande Ceinture (ordinaire et complémentaire) et l'autoroute A3. Photo prise en direction de Paris depuis le pont de l'avenue Jules-Ferry à Bondy.

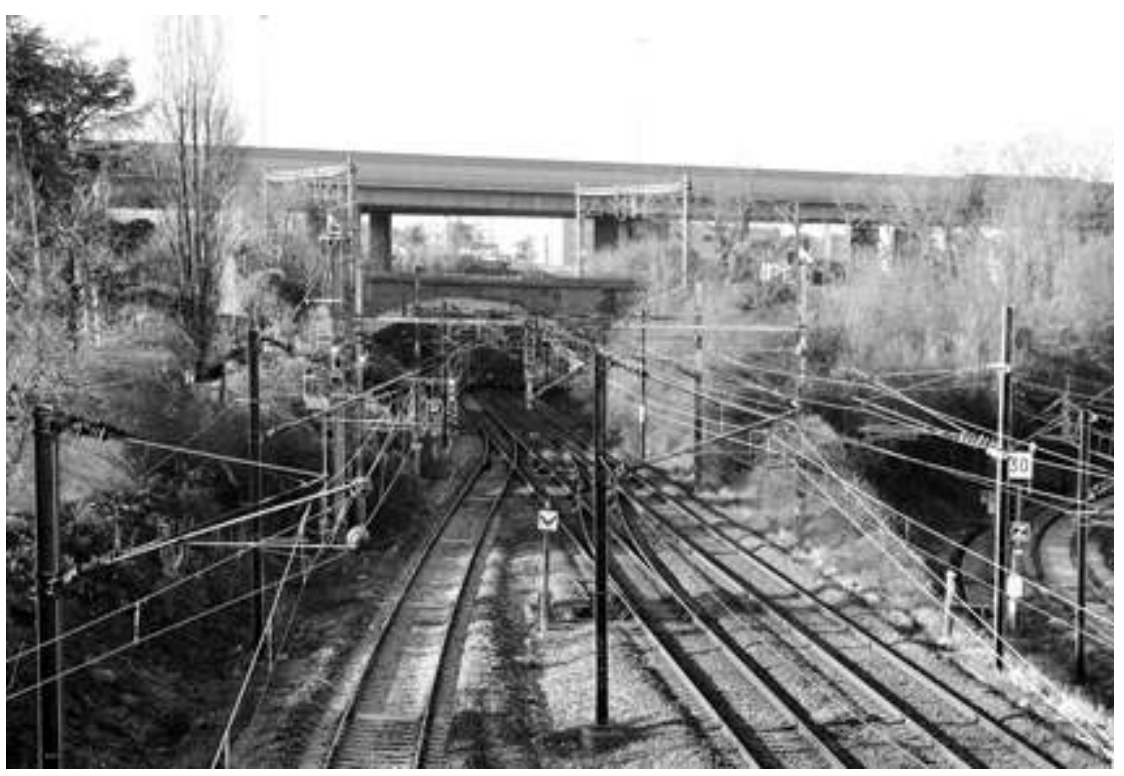

(c) G. Jigaudon, CNAM / Département de la Seine-Saint-Denis. 
Un dernier volet de nos études est la dimension mémorielle du patrimoine ferroviaire, avec l'exemple de la gare de Bobigny, gare de déportation depuis le camp de Drancy à partir de 1943 après Le Bourget et qui a été protégée en 2005 au titre des monuments historiques pour ce motif. La ville de Bobigny développe un projet d'étude et de valorisation de ce site au titre de lieu de mémoire (fig. 14).

Figure 14. La gare de Bobigny Grande Ceinture.

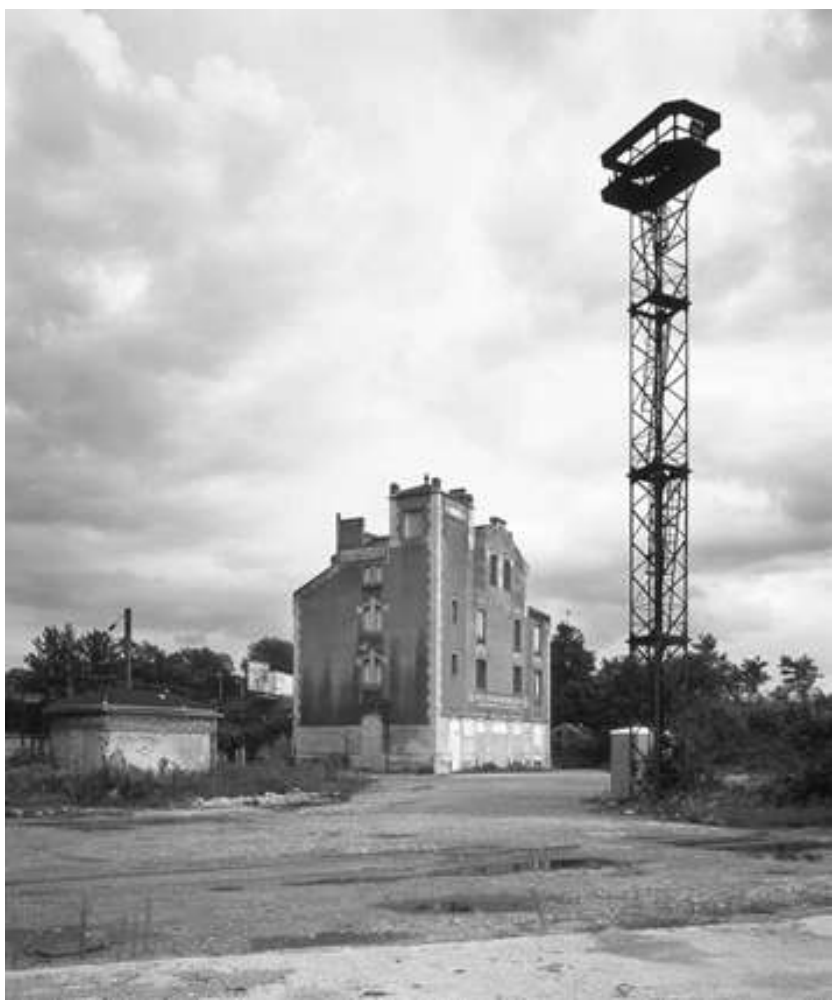

(c) L. Desmoulins, Département de la Seine-Saint-Denis.

Les directions de recherche sont donc multiples comme sont nombreuses les difficultés qu'elles soulèvent. Quant aux questions qu'elles posent, nous en retenons deux. La première est la méthode d'étude à appliquer à un patrimoine linéaire sur une portion de territoire : comment dépasser les limites géographiques et institutionnelles? Pour obtenir un résultat équivalent à celui de l'étude de la ligne Paris-Le Havre, je proposerais un groupe de travail à l'échelle de l'île-de-France qui facilite l'étude d'un territoire qui va de Paris jusqu'à la lointaine banlieue. La seconde question est celle de la reconnaissance de ce patrimoine technique parfois difficile à comprendre : comment promouvoir la notion de culture matérielle, de culture technique, quelles sont les conditions de cette reconnaissance?

\section{Anne Hecker}

Dans le cadre des recherches sur le patrimoine ferroviaire, un domaine est trop rarement abordé, celui de l'infrastructure. Il est vrai que la voie ferrée proprement dite, et surtout l'emprise qui en reste une fois la circulation ferroviaire abandonnée, sont rarement évoquées en termes de patrimoine. En effet, souvent, lorsque l'infrastructure ne constitue pas un élément patrimonial fort, par la présence d'un ouvrage d'art exceptionnel par exemple, ou par une inscription dans le paysage 
particulièrement remarquable, elle a tendance à être considérée comme un élément patrimonial mineur et à demeurer totalement méconnue.

Pourquoi parler de patrimoine linéaire dans de telles conditions? D'abord, parce qu'un grand nombre de voies ferrées ont été désaffectées. On estime qu'environ $15000 \mathrm{~km}$ d'infrastructures ferrées dédiées aux voyageurs ont ainsi disparu en quelques décennies. Ces voies ferrées d'au moins quelques dizaines de kilomètres, pour la plupart, et qui souvent étaient interconnectées, constituaient un capital foncier linéaire peu large, certes, mais particulièrement étendu. Son aspect patrimonial se traduit par des qualités très variées. Tout d'abord, un profil en long extrêmement favorable, dans lequel on ne trouve ni rampes fortes, ni courbes trop serrées, etc. Ce patrimoine relève bien entendu du domaine des innovations techniques, par la construction des ouvrages d'art, des gares, des déblais, des remblais, etc. C'est bien sûr un patrimoine porteur de la mémoire ouvrière, de la mémoire cheminote. C'est aussi un patrimoine dans un domaine moins attendu, pourtant très à la mode, qui est l'écologie, avec la présence de végétaux spécifiques tout au long des voies de chemin de fer, ce qui était déjà vrai lorsque ces voies étaient en service. Malgré des désherbages réguliers, on constate la présence, notamment sur les talus de chemin de fer, de plantes peu répandues, de plantes exogènes, et cela se vérifie plus encore après l'arrêt de la circulation ferroviaire, quand ces plantes se développent davantage. On constate également l'apparition de nouvelles espèces qui colonisent principalement les espaces abandonnés. Les voies ferrées abandonnées sont des vecteurs de circulation de la faune et de la flore assez remarquables, qui permettent de constituer un relais des réseaux de haies qui ont tendance à disparaître en milieu rural et qui favorisent les échanges entre les espèces. Ce sont là des éléments patrimoniaux peu connus mais qui méritent d'être pris en compte.

34 Pourquoi ce patrimoine est-il méconnu? D’abord, parce que, après son abandon, une emprise ferroviaire passe dans les mains du service des domaines et, pendant des décennies, elle a été vendue par tronçons. Généralement, les infrastructures étaient vendues aux personnes qui en voulaient bien et il n'était pas toujours évident pour la SNCF de trouver des acquéreurs. Souvent, ce patrimoine a été tronçonné et cédé aux mairies traversées par ces emprises, qui elles-mêmes les ont tronçonnées à nouveau, en vendant chaque morceau à ses riverains, ce qui a conduit à un véritable «mitage » de ce capital linéaire, pour reprendre ce terme qu'on utilise habituellement pour la banlieue. Ce n'est plus toujours vrai aujourd'hui. Malgré tout, pendant des décennies, c'est le mode de cession du capital linéaire qui a prévalu, ce qui a conduit à sa disparition, ou du moins à la disparition d'une grande partie d'un patrimoine qui a été transformé en jardins, en rues de lotissement, en parcs pour animaux, en champs de blé, quand il n'est pas tout simplement retourné à la friche en milieu forestier ou en milieu rural. On a donc assisté à la disparition d'une grande partie de ce patrimoine qui était pourtant chèrement construit et chèrement acquis.

Ce patrimoine est également méconnu parce que rares sont les personnes qui s'en préoccupent réellement. Lorsqu'on évoque le patrimoine ferroviaire, on pense bien sûr au patrimoine bâti, aux bâtiments remarquables comme certaines gares ou même d'autres bâtiments peut-être moins exceptionnels, mais malgré tout reconnus; on pense également aux ouvrages d'art, à la mémoire cheminote, aux archives du monde du travail. Pour autant, le fondement même de ce patrimoine, sans lequel la circulation ferroviaire aurait été impossible, est rarement pris en compte. Il constitue pourtant 
l'infrastructure à proprement parler, surtout une fois qu'elle a disparu et qu'elle est sortie du domaine ferroviaire.

36 C'est en effet un élément jugé banal lorsqu'il n'est pas ponctué par de grands ouvrages d'art ou de belles gares. Somme toute, à quoi bon conserver quelque chose de banal ? À quoi bon chercher à en dresser l'inventaire ? À cela, on peut rétorquer qu'il s'agit d'un élément de mémoire. Ça l'est pour toutes les raisons que j'ai mentionnées plus haut, au même titre qu'une gare ou qu'un ouvrage d'art. C'est également un élément qui participe de la mémoire d'un lieu, un élément du paysage qu'il contribue à modeler lors de sa réalisation. Il suffit de penser par exemple à tous les remous auxquels on a assisté lors de la mise en place de la nouvelle ligne de chemin de fer du TGV Est: même si la nouvelle infrastructure était relativement étroite - on ne parlait pas de faisceau - elle a malgré tout provoqué de nombreuses difficultés localement. Lorsqu'une infrastructure est abandonnée, le paysage évolue, une friche prend la place d'une liaison de chemin de fer, elle est considérée comme une verrue dans le paysage, c'est un élément qui participe du paysage. Or elle a également participé au façonnement du paysage, tel qu'on le constate aujourd'hui, notamment en favorisant les déplacements des hommes et des marchandises. Un exemple, parmi tant d'autres, est la disparition de la vigne dans les paysages ruraux les moins favorables à cette culture. En Lorraine, par exemple, des coteaux bien exposés étaient couverts de vignes qui ont généralement disparu lors de l'apparition des lignes de chemin de fer et de la multiplication des transports relativement bon marché qui permettaient de faire venir des vins de meilleure qualité à des tarifs supportables, ce qui a progressivement contribué à la disparition du vignoble. Ce facteur n'est pas la seule explication, mais c'est un élément d'explication de la mémoire du paysage et de son évolution.

Écarter ces témoins revient à perdre cette mémoire, une partie de l'explication de l'évolution du paysage, et c'est dommage. Pour autant, faut-il tout conserver ? Et peuton tout conserver? Cette question mérite d'être posée. A priori, la réponse est non, ne serait-ce que parce que toutes ces emprises d'aujourd'hui sont, pour la plupart, tellement délabrées qu'il serait impossible de les sauvegarder. Beaucoup ont totalement disparu, je pense bien sûr aux voies ferrées abandonnées depuis de nombreuses années. Elles ont fréquemment été englobées dans leur environnement, qu'il soit rural ou urbain. Souvent, la demande d'espace est forte - je pense notamment au milieu urbain et périurbain - et entraîne une assimilation très prompte de cet espace disponible, nous en avons parlé à propos de la région parisienne, mais c'est aussi vrai dans tous les milieux urbains où règne une certaine pression foncière. En milieu rural, c'est plutôt la volonté de faire disparaître un élément qui constitue une gêne ou un obstacle aux communications, notamment dans les champs ou dans les espaces en cours de remembrement, où un déblai ou un remblai constitue un tel obstacle.

Face à cette situation, il est assez difficile d'envisager la conservation de ces milliers de kilomètres de voies ferrées. Par ailleurs, la sauvegarde a un coût : il faut racheter, il faut transformer, il faut entretenir. Cela mérite réflexion, surtout sur le devenir de ces éléments sauvegardés, sur leur utilisation. Quel en est l'intérêt, et sous quelle forme les sauvegarder ? Récemment, dans ce domaine, on a assisté à une évolution de l'opinion publique concernant les voies ferrées désaffectées, de deux façons radicalement différentes. D'une part avec le retour du train, vous mentionniez à juste titre que le train était redevenu un mode de déplacement intéressant, ce n'est plus quelque chose de dépassé, de passéiste. On assiste donc à des demandes de réouverture de voies de 
chemin de fer. Certaines ont abouti et obtiennent de très bons résultats. Cela bien sûr n'est possible que lorsque le tracé existe encore et que l'emprise peut être remise en état rapidement. Un autre élément complètement différent de valorisation des voies ferrées est apparu antérieurement, c'est la naissance des notions de vélo-route et de voie verte, ces itinéraires destinés aux circulations non motorisées, soit dans un objectif de déplacement, notamment en milieu urbain et périurbain, soit dans un objectif de loisir ou de tourisme. Ces notions de vélo-route et de voie verte sont nées aux États-Unis et sont arrivées en Europe par le biais, d'une part du Royaume-Uni, d'autre part de la Belgique, qui ont été des précurseurs dans ce domaine. Leur exemple est suivi aujourd'hui par l'Espagne qui développe ce réseau, et par des projets paneuropéens.

Pour certains, il s'agit d'une véritable volonté de sauvegarder le patrimoine ferroviaire. Je pense par exemple à la Belgique, particulièrement impliquée dans ce secteur. Elle va transformer en priorité ses infrastructures ferroviaires, ainsi que des voies de halage, les relier entre elles, les valoriser, leur attribuer une signalétique, une signalisation. Une telle orientation présente un intérêt pour le patrimoine ferroviaire puisqu'elle permet de préserver le capital linéaire en le conservant, sinon dans sa totalité, du moins dans la plus large partie possible. Parfois, elle permet également de le valoriser en termes de transport, puisqu'il retrouve des fonctions utilitaires en milieu urbain et périurbain pour pallier une moindre utilisation de la voiture. La plupart du temps, malgré tout, ces aménagements ont une vocation de loisir ou de tourisme. C'est une valorisation de qualité du capital linéaire et du profil en long qui met à profit cette absence de rampes, de pentes, très agréable lorsqu'on n'est pas motorisé. Cela permet également de conserver l'accessibilité des centres-villes, de manière totalement indépendante de la circulation motorisée. Cette préservation est donc un atout important pour les secteurs qui accueillent ces voies vertes. Elle leur permet, en outre, de valoriser les espaces concernés en assurant la disparition d'une verrue paysagère, lorsqu'il s'agit d'une emprise qui avait été particulièrement dégradée, et en réintégrant à la ville un élément qui faisait partie de son paysage. C'est également un apport aux pratiques touristiques régionales avec pour certains secteurs des retombées économiques très fortes. Je pense, par exemple, à une voie longeant le Danube, même s'il s'agit d'une voie de halage et non d'une voie ferrée, ou encore à Givry-Cluny qui est un exemple ferroviaire.

Pour autant, cette valorisation patrimoniale ferroviaire reste imparfaite. En effet, elle existe rarement, hormis pour quelques cas que j'ai cités comme la Belgique, l'Espagne, ou les États-Unis. Pour le reste de l'Europe, c'est principalement une forme d'aliénation du patrimoine ferroviaire. En effet, les projets ne relèvent pas du tout de la préservation de la mémoire ferroviaire. C'est par exemple le cas de la vision très pragmatique de projets en Grande-Bretagne, ou des projets paneuropéens qui vont tracer sur le papier un réseau qui va soit correspondre à des besoins de déplacement, c'est le cas au Royaume-Uni, soit à des trajets sous-tendus par de grandes idées, comme la voie "Charles le Téméraire ", par exemple, qui sont des projets porteurs d'un certain sens, mais totalement détachés de la préservation du patrimoine ferroviaire, du patrimoine des voies d'eau intérieure ou autre patrimoine viaire. Ensuite, sur le terrain, une seconde étape consiste à rechercher quelles sont les infrastructures qui pourront être utilisées et comment réaliser ce trajet que l'on a dessiné. Dès lors, les voies ferrées sont intégrées dans ce genre de projets mais, finalement, uniquement lorsqu'elles se trouvent, presque par hasard, bien situées par rapport au trajet fixé. On se retrouve 
ainsi avec des emprises qui ont été déjà réaménagées, mais qui sont exclues de ces projets, parce qu'elles sont écartées de vingt ou trente kilomètres.

Par ailleurs, étant donné la façon dont ces projets sont menés, on perd totalement l'identification de l'emprise des anciennes voies ferrées puisque, lorsqu'elles sont réutilisées, elles sont incluses dans un grand projet, dans de vastes routes qui font plusieurs centaines de kilomètres et qu'elles y sont finalement noyées. Elles sont banalisées, elles ne sont pas plus reconnaissables en tant que voies ferrées que ne peut l'être un chemin ou un sentier, ou une route calme qui a été intégrée dans ce projet.

Dès lors, on peut se demander si cette forme de valorisation est forcément la meilleure en termes de patrimoine ferroviaire. C'est certes un élément qui mérite d'exister dans la mesure où il permet de sauver un certain nombre d'emprises ferroviaires, ce qui n'est pas négligeable; peut-être faudrait-il cependant faire évoluer les choses de manière à pouvoir y intégrer, comme en Belgique, une notion de préservation de l'image du passé ferroviaire. Peut-être est-il possible également d'inventer une valorisation complémentaire à celle-ci, de manière à pouvoir réintégrer les voies ferrées qui en sont exclues et de trouver une solution pertinente au regard du patrimoine ferroviaire. C'est donc l'une de mes questions.

Sauvegarder, oui, mais pour quoi faire ? Au-delà de ce concept des vélos-routes et des voies vertes, que peut-on faire des voies ferrées désaffectées qui n'entreraient pas dans ce cadre ? Peut-on faire évoluer ce concept ? L'évolution est possible, mais encore fautil se faire entendre, notamment dans les projets paneuropéens, or il est difficile de faire entendre une voix discordante. Proposer d'autres solutions d'aménagement, pourquoi pas? Mais lesquelles? Et comment les mettre en place ? Et, là encore, comment se faire entendre ? Par ailleurs, quel critère permettrait de juger de la nécessité et de l'utilité de préserver ou non une infrastructure relativement banale au regard des ouvrages d'art et des gares, des monuments? Doit-on la juger sur des qualités techniques, sur son intégration paysagère, sur l'existence d'un projet, sur son utilité dans le cadre d'un projet de développement économique ou touristique? En d'autres termes, faire de ce patrimoine quelque chose d'efficace? D'autres critères pourraient-ils être définis ? Là encore, comment arriver à la connaissance de ce patrimoine et comment imposer finalement cette connaissance?

\section{Jean-François Belhoste}

Je vais surtout vous parler de mon expérience de vingt années au sein de l'Inventaire général.

Revenons sur la question, déjà abordée, du rôle de la protection au titre des monuments historiques et du ministère de la Culture dans l'orientation des recherches et sur l'apport que ces préoccupations patrimoniales ont pu représenter pour une recherche plus classique en matière de chemins de fer. Les préoccupations patrimoniales ferroviaires étaient totalement liées à celles qui ont vu le jour dans les mêmes années 1980 à propos du patrimoine industriel avec la création, en 1983, de la cellule du patrimoine industriel au sein de la sous-direction de l'Inventaire. L'apport de cette nouvelle façon de rapprocher le patrimoine de l'histoire, c'est évidemment l'approche archéologique. C'est pour cela qu'on parle d'archéologie industrielle : elle consiste à partir des objets matériels pour voir ce qu'ils peuvent nous dire et à croiser les informations tirées de l'observation des choses avec ce que nous apprennent les archives. 
Je rappelle en bref comment, en matière ferroviaire, la question du patrimoine et de la protection s'est posée. D'abord, en 1974, une série de treize ou quatorze protections, liées à la prise en compte du patrimoine des $\mathrm{XIX}^{\mathrm{e}}$ et $\mathrm{XX}^{\mathrm{e}}$ siècles; en 1984, une grande série de protections, initiée par le directeur du patrimoine de l'époque, Jean-Pierre Weiss, dans le cadre d'une relation et d'un accord entre le ministre des Transports Charles Fiterman et Jack Lang, donc une démarche volontariste; ensuite, toute une série de protections, souvent menées au coup par coup, qui ont été entreprises au sein des services de l'inventaire général en charge des études systématiques d'inventaire, dans le cadre de réflexions sur le patrimoine industriel et dans celui d'un certain nombre de programmes d'inventaire thématique. Cela a été d'abord, bien entendu, la question des gares. Ces travaux se sont réalisés, entre autres, en connexion avec les écoles d'architecture et avec le travail qui a été déjà plusieurs fois évoqué de Karen Bowie. Lié au fait que ce type de monuments avait été sélectionné en grande quantité dans les premières listes, un travail sur les viaducs, sur les grands ouvrages d'art a été réalisé, en particulier les opérations d'inventaire et d'étude des viaducs d'Auvergne menées dans les années 1990. Mais aussi, concernant des éléments plus méconnus, fort intéressants et, cette fois-ci, liés directement aux travaux d'archéologie industrielle, des protections et des travaux de recherche sur les lignes de la région de Saint-Étienne : Saint-Étienne-Andrézieux et Saint-Étienne-Lyon, des lignes qui au départ avaient un caractère fortement industriel, étant liées à l'industrie minière et métallurgique. Dans un sens inverse de celui qui partait de la protection patrimoniale pour aller vers des études plus larges d'inventaire et des réflexions historiques sur la constitution de ce patrimoine, ce travail a abouti à des protections qu'on a un peu oubliées mais que je trouve tout à fait intéressantes, celles du tunnel de Couzon et du pont de Chevignon. Le tunnel de Couzon est un tunnel encore en activité pour le TGV entre Saint-Étienne et Lyon qui représente une partie du démarrage des chemins de fer, avec l'œuvre des frères Seguin dans les années 1830 . Pour terminer cette série de travaux réalisés dans le cadre de l'inventaire général, une opération dont on a déjà parlé et qui, elle, s'est faite de façon totalement indépendante des préoccupations de protection, est l'étude du réseau Ouest, plus précisément de la ligne Paris-Rouen-Le Havre qui a donné lieu en 2006 à une publication dans la collection des Images du patrimoine et à une série d'expositions. Il est intéressant de noter que, à partir de cette réflexion sur le réseau Ouest, des travaux ont parallèlement été menés sur la gare Saint-Lazare, sur la gare des Batignolles, ces derniers réalisés en relation avec la Commission du Vieux-Paris, et des réflexions historiques sont encore en cours sur le chemin de fer Le Pecq-SaintGermain-en-Laye, c'est-à-dire ce chemin de fer tout à fait extraordinaire qui à ses débuts adoptait la technique du chemin de fer atmosphérique. Ce sujet a été l'occasion d'échanges passionnants entre la Grande-Bretagne et la France et toute une série d'innovations ont résulté de cette réflexion sur ce nouveau mode de propulsion*. Il en reste des éléments patrimoniaux : les ponts sur la Seine, le tunnel pour accéder à SaintGermain-en-Laye, mais, un peu comme pour la gare des Batignolles, ces travaux d'histoire sur le patrimoine n'ont pas donné lieu à des protections. Une sorte de renversement s'est produit par rapport à la situation initiale où des politiques de protection incitaient des politiques de recherche en matière patrimoniale. Là, le travail, ou plutôt l'ambition qui, a priori, devait présider à ces travaux de réflexion sur le patrimoine, à savoir aboutir à une protection, à une mise en valeur, n'a pas pu être satisfaite. 
Quels sont finalement les acquis, en matière de recherches historiques, de ces différents types de travaux qui sont venus abonder la recherche historique plus large avec leurs spécificités et qui, encore une fois, résultent du fait qu'on part de l'objet technique, des objets matériels, qu'on les croise avec les archives dans une perspective inverse de celle de la recherche historique classique? D'une part, des avancées sur les techniques de construction, en particulier sur l'usage du métal dans la réalisation du matériel ferroviaire au sens large, qu'il soit fixe ou roulant. D'autre part, cette perspective, progressivement prise en compte, qui met sur le même plan toute une série d'éléments patrimoniaux destinés à être rassemblés dans un ensemble qui est celui du réseau, celui aussi des coulisses de l'exploitation. On va pouvoir aller éventuellement jusqu'aux usines de fabrication du matériel. Et tout cela, dans un programme qui n'est pas forcément toujours réalisé, mais qui a le mérite de poser le problème en ce sens.

Quelles questions peut-on se poser à partir de ces acquis qui laissent encore ouverts d'immenses champs libres? D'une part un problème de chronologie, avec des écarts entre ces préoccupations patrimoniales, qui partent très souvent d'éléments opérationnels aujourd'hui, et la recherche historique large qui, elle, veut couvrir toute la chronologie ; le matériel roulant qui ne fait pas l'objet de cette table ronde, mais qui appartient au champ du patrimoine, concerne surtout le $\mathrm{xx}^{\mathrm{e}}$ siècle, alors que beaucoup de travaux d'histoire vont porter sur la mise au point des prototypes ou des premières machines locomotives, celles, par exemple, de la deuxième moitié du xix siècle. En revanche, au départ, les monuments, les bâtiments, les gares vont être protégés surtout pour leur ancienneté, alors qu'un problème se pose pour les éléments plus récents comme dans une approche proprement «monuments historiques». Donc des décalages, selon les champs patrimoniaux, et des hiatus avec les champs de la recherche historique plus classique.

Je poserai également le problème de l'histoire des techniques. Le rapport entre l'histoire des techniques et le patrimoine est une question assez difficile, parce que François Loyer l'a dit pour commencer, la technique a eu du mal à s'imposer comme relevant de l'histoire et le patrimoine technique ou les œuvres techniques à s'imposer comme ayant une valeur patrimoniale. C'est précisément dans ces années 1970-1980 que, petit à petit, le patrimoine technique a pris rang dans le patrimoine industriel et au sein du patrimoine au sens large, en particulier par le fait qu'il a été reconnu comme monument historique. Je vous rappelle que, de ce point de vue, 1986 est l'année où a été installée au sein de la Commission nationale des Monuments historiques une section chargée du patrimoine industriel scientifique et technique.

Il n'empêche que l'ingénieur, lui, n'est pas encore bien étudié et en tout cas considéré comme un auteur patrimonial. Je crois que l'on peut dire que l'essentiel de ce qui a pu être protégé au titre des monuments historiques l'a été parce que, derrière l'œuvre, il y avait un grand architecte. Il n'y a qu'un seul exemple d'ingénieur dont on a pu classer ou inscrire les œuvres parce qu'il était ingénieur, c'est Gustave Eiffel, avec les premières protections, en 1965 , le 8 décembre, des deux viaducs de Neuvial et de Rouzat, après celui de Garabit, le 14 septembre. Ce qui est intéressant dans ce cas-là, c'est qu'il existe en réalité quatre viaducs semblables à valeur patrimoniale, mais seuls deux, ceux d'Eiffel, ont été protégés, mais pas ceux réalisés par l'entreprise Cail, pourtant absolument jumeaux.

51 J'entendais tout à l'heure parler de la gare où nous sommes, la gare d'Orsay. L'ingénieur qui a conçu cette gare, Eugène Bertrand de Fontviolant (1861-1954), est un des plus 
grands praticiens et théoriciens de la résistance des matériaux. Je ne pense pas que ce point ait été utilisé pour sauver le bâtiment et en faire ce qu'il est devenu. Le problème de l'ingénieur reste entier. J'entendais tout à l'heure François Loyer mentionner la difficulté des rapports entre les architectes des bâtiments de France et les architectes praticiens. Dans l'optique d'un ingénieur, il n'y a pas d'ingénieurs des bâtiments de France, ou d'ingénieurs en chef des monuments historiques, le problème ne se pose même pas. Les ingénieurs, en tant que corporation, ne défendent absolument pas leurs œuvres et, en matière ferroviaire, c'est un réel souci. Voici une question: celle de savoir comment les ingénieurs pourraient s'intéresser davantage à leur patrimoine pour faire en sorte qu'il soit préservé et valorisé.

52 Le dernier point, sur lequel je voudrais conclure, c'est celui de la réorganisation complète de la gestion du patrimoine en France actuellement, notamment au sein du ministère de la Culture. L'inventaire général était jusqu'en 2004 un service d'État relevant de la direction de l'Architecture et du Patrimoine et travaillant en relation avec les Monuments historiques en qualité de service d'études, mais séparément, avec pour ambition de réaliser un inventaire qui puisse par la suite servir à sélectionner les éléments les plus remarquables qui seraient protégés à ce titre. D'où le fait qu'il y ait eu des opérations d'étude du patrimoine ferroviaire liées à la politique de protection. Ces services de l'inventaire relèvent maintenant des régions et non plus de l'État. Ils peuvent aussi relever des départements, avec des services départementaux du patrimoine particulièrement actifs comme ceux de Seine-Saint-Denis et de Seine-etMarne. Dans le même temps, le nombre de protections en général et industrielles et ferroviaires en particulier, que ce soit l'inscription ou le classement, ne cesse de diminuer. La configuration est totalement nouvelle, mais pas forcément mauvaise. En tout cas, il faut en prendre acte, à un moment où la loi sur les monuments historiques devient de moins en moins intéressante pour enclencher des processus de protection et de mise en valeur et où on peut utiliser bien davantage les lois d'urbanisme que sont les ZPPAUP (zones de protection du patrimoine architectural, urbain et paysager) et les plans locaux d'urbanisme. Le paysage est différent et, sans doute, même si je ne connais pas l'état des forces, la politique de la SNCF a-t-elle changé par rapport à la grande opération de protection du patrimoine ferroviaire de 1984, RFF est apparu.

53 Comment donc avec ces différents acteurs, avec aussi une augmentation des travaux sur ces thèmes dans les universités et dans les écoles d'architecture, le cadre général de la recherche va-t-il se recomposer en prenant en compte les enjeux et défis qui sont posés aujourd'hui au patrimoine?

Intervention de madame Catherine Bergeal, sous-directrice de la Nature et des Paysages, ministère de l'Écologie, du Développement et de l'Aménagement durables, présidente de la séance, dans le débat.

\section{Catherine Bergeal}

En tant que sous-directrice au ministère du Développement et de l'Aménagement durables - le pluriel de l'adjectif étant au cœur de ce que nous allons partager ensemble -, je suis très heureuse de participer au vingtième anniversaire de l'Association pour l'histoire des chemins de fer en France, un partenaire important pour nous depuis plusieurs années, qui s'inscrit parfaitement dans l'actualité de notre grand ministère. Je pense en effet que, pour être vraiment durable et optimal par rapport au territoire, un aménagement nécessite une connaissance de ce territoire, de ses paysages. Nous allons 
donc débattre de la façon dont nous pouvons faire reconnaître un patrimoine partagé dans l'aménagement durable de nos territoires.

Nous sommes vraiment dans une période passionnante, mais totalement paradoxale, à propos de laquelle, je pense, mes fonctions me permettent de témoigner. On observe, et sur ce point, je rejoins $\mathrm{M}$. Loyer, un changement de cap complet en ce qui concerne le partage des politiques du patrimoine. Je m'occupe au ministère de l'Écologie du patrimoine, de la protection des sites. Mais j'ai une histoire, je suis architecte, je suis urbaniste. Il y a vingt ans, en matière d'urbanisme dans un État décentralisé, je disais qu'il faudrait bien trente ans pour qu'on se réapproprie le paysage, qu'on reparle $d u$ patrimoine, des territoires. Et je vis une période où l'on voit que les élus, la société, expriment très fortement cette nécessité de reconnaître les ressources, les singularités de l'histoire des territoires. Le patrimoine est devenu un débat réel dans la société et on s'en réjouit. Je pense que nous sommes nombreux à être très satisfaits des progrès de la recherche, de la qualité de l'enseignement, de petits bonheurs quotidiens dans ce domaine. Mais au cours d'une même période, d'une même journée, voire d'une même heure, on se heurte à des désillusions. Ce quotidien nous amène à mettre en place des politiques publiques qui lui correspondent. Le patrimoine, ça se révèle d'abord, ça se connaît, ça s'explique, ça se remet en perspective, pour qu'effectivement les élus et les décideurs en soient conscients. Ce n'est pas une évidence donnée au départ. On décide vite, il faut arbitrer en permanence des intérêts contradictoires, il faut être extrêmement précis et extrêmement percutant quand une valeur dépasse un intérêt purement local et présente un sens collectif qu'il faut défendre. On ne fait pas se succéder à la défense contre les Vandales une période bénie de consensus. Non, il faut en permanence faire connaître le patrimoine pour pouvoir le partager, tout simplement pour pouvoir en débattre. Le patrimoine doit être objet de débat. Il faut par conséquent être vigilant parce que rien n'est jamais acquis, même quand un site est inscrit sur la liste du Patrimoine mondial. Je peux vous assurer qu'il n'est pas à l'abri des bêtises quotidiennes, alors que tout le monde s'accorde sur sa valeur : personne ne veut porter atteinte à une valeur d'intérêt mondial mais on doit néanmoins mener des petits combats quotidiens contre des actions dans lesquelles tout le monde a sa part de responsabilité.

Ensuite, il faut faire du projet, créer un projet collectif qui va concilier des projets différents mais qu'on a l'ardente obligation de concilier et d'enrichir. Les projets sont enrichis par cette connaissance et prennent une dimension bien plus intéressante s'ils peuvent être élargis à un projet social avec des matériaux réellement intéressants et à moindre coût énergétique, exigences parfaitement conciliables. Et c'est même parfois le fil directeur d'un bon projet. Il faut se nourrir de ce patrimoine, mais aussi le réinterpréter, utiliser des matériaux d'aujourd'hui, les faire entrer dans la modernité. Mais nos territoires, nos histoires, doivent nourrir les projets, c'est véritablement le premier appel que j'ai envie de lancer. Je vous donnerai un autre exemple de contradiction. D'un côté, une convention européenne du paysage entre en vigueur en France assortie d'une circulaire dont j'ai fait la promotion le $1^{\text {er }}$ mars 2007, qui propose qu'il y ait dans tous les départements français, et tous les ans, au moins une journée où l'on parle des paysages du département, journée structurée selon cette convention européenne. Plusieurs États l'ont déjà ratifiée, mais où va-t-on trouver de la connaissance, que sait-on des paysages du département, quels sont les enjeux principaux de la journée? Qui doit-on y inviter? Les associations ont là un rôle ainsi que les professionnels du champ, les gens qui connaissent les territoires, qui travaillent 
sur ces sujets. Il a été décidé le $1^{\text {er }}$ mars 2007 qu'il fallait parler des paysages, échanger, confronter et pas simplement dire : «Parlons-en une fois et oublions-les. » C'est très important. Il est vrai que la logique sectorielle vise toujours à simplifier la donne; on oppose les choses. On dit : « Il faut choisir, Monsieur l'élu : ou vous voulez du logement social, ou vous voulez du patrimoine. » Je crois pourtant qu'il est possible d'avoir les deux, qu'il faut être gourmand. Il est impératif de débattre de cela, d'être vigilant.

Deuxième thème, le Grenelle de l'environnement. Le sujet que vous signalez est mon quotidien. Quand vous parlez de haute qualité environnementale, de choix énergétique, eh bien, cela veut dire le choix des éoliennes. Je ne suis pas contre les éoliennes, mais il faut un dialogue qui est un dialogue légitime. Les ministres qui se sont succédé ont toujours dit: "Oui aux éoliennes, mais pas n'importe où ni n'importe comment. » Il faut effectivement faire place à toutes les solutions énergétiques, mais la ressource du territoire est rare. La planète bleue, tout le monde a compris qu'elle était limitée, mais c'est aussi le cas de notre périphérie parisienne, pressée par les besoins à satisfaire pour accueillir des logements, des activités, pour accompagner les mutations nécessaires. La ressource première, c'est le territoire et elle est très rare et très riche d'histoire. Le paysage aujourd'hui c'est vraiment d'abord de l'histoire qu'il faut savoir retraduire - que garder, que détruire, qu'avons-nous déjà laissé partir...? - Or la structuration du territoire par les réseaux d'infrastructure est vraiment un fil directeur dont la compréhension est primordiale afin d'éviter, de temps en temps, de refaire des erreurs qui coûteraient cher en termes énergétiques, sociaux, et conduiraient à une moins bonne organisation spatiale de nos territoires.

\section{NOTES}

*. Voir en dernier lieu : Paul Smith, «Les chemins de fer atmosphériques », In-Situ, n 10 (19 mai 2009), [139 pages], en ligne : www.revue.inventaire.culture.gouv.fr

1. Goulven Guilcher, "Les guides de chemin de fer: pratiques anglaises et françaises », in François Moureau et M.-N. Polino (dir.), Écritures du chemin de fer, Paris, Klincksieck, 1996, p. 23-36.

2. François Bon, Paysage Fer, Lagrasse, Éditions Verdier, janvier 2000 ( $1^{\text {re }}$ édition); Paysage Fer, le film, documentaire de création, 52', production Arte et Imagine, réalisation Fabrice Cazeneuve, images Pierre Bourgeois et Fabrice Cazeneuve, texte François Bon, montage Jean-Pierre Bloc, première diffusion sur Arte, décembre 2003; photos de tournage et documents sur le site: http://www.tierslivre.net/livres/paysfer.html (consulté le 18 novembre 2008). [N.d.l.R.]

3. Antoine Picon, L'Invention de l'ingénieur moderne. L'École des ponts et chaussées, 1747-1851, Paris, 1992.

4. "Les ponts et chaussées et les Bouches-du-Rhône ", thèse de doctorat, université de Provence, 1991.

5. Hélène Bocard, et alii, De Paris à la mer. La ligne de chemin de fer Paris-Rouen-Le Havre, Paris, coll. «Images du patrimoine », n²39, 2006. 


\section{RÉSUMÉS}

Animé par Karen Bowie, ce débat doit permettre à chacun des participants de réagir aux propositions de nouvelles recherches faites par le comité scientifique de l'AHICF dans le domaine du patrimoine ferroviaire, de l'architecture, de l'urbanisme et des paysages. Il réunit François Loyer, directeur de recherche au CNRS, Evelyne Lohr, conservateur chargée de l'Inventaire au Service du patrimoine culturel du Département de Seine-Saint-Denis, Anne Hecker, géographe, maître de conférences à l'université de Nancy II, Jean-François Belhoste, directeur d'études à l'École pratique des hautes études, et Catherine Bergeal, sous-directrice de la Nature et des Paysages au ministère de l'Écologie.

En introduction, F. Loyer remarque que le chemin de fer, loin de devenir obsolète comme on pouvait le penser dans les années 1960, est devenu le vecteur de la mobilité moderne. La notion de patrimoine se définit alors comme un passage de relais entre deux époques et non comme la nostalgie d'une culture matérielle disparue. Si patrimoine et architecture ont longtemps été antinomiques, comme urbanisme et paysage, on constate aujourd'hui des rapprochements qui renouvellent ces notions. Le débat devra donc inclure les paysages en rapport avec les chemins de fer, les abords du bâti, l'architecture des bâtiments et le design, « architecture sans site ».

Chacun des participants expose les perspectives de son domaine de recherche.

Evelyne Lohr analyse le rôle des emprises ferroviaires dans le territoire du département de la Seine-Saint-Denis concerné par de très importantes mutations. Elle montre comment, et avec quelles méthodes d'analyse rationnelle et documentée, le Service du patrimoine culturel contribue à la compréhension de ce territoire complexe et lui apporte une cohérence, en comprenant le patrimoine comme un moteur d'évolution et non un objet de nostalgie. Les directions de recherche sont multiples: impact des réseaux sur le territoire, chemin de fer comme facteur d'urbanisation, réutilisation de son patrimoine, paysages créés par le chemin de fer... Les problèmes posés par l'inventaire le sont autant, comme la méthode d'étude à appliquer à un patrimoine linéaire sur une portion de territoire, ou la reconnaissance d'un patrimoine technique parfois difficile à comprendre.

Anne Hecker aborde, en termes de patrimoine, l'infrastructure ferroviaire, la voie ferrée et son emprise. Patrimoine linéaire étendu et aux caractères bien marqués (profil, tracé, modes de construction innovants, support de mémoire ouvrière et locale, conservatoire pour une faune et une flore remarquables), il reste méconnu. Pourtant, la question de sa conservation partielle, comme témoin et explication de l'évolution du paysage, doit être posée, dans la double perspective de sa valorisation par la réouverture de voies au trafic ou par la transformation de voies déferrées en équipements touristiques, vélos-routes et voies vertes. Cette dernière n'assure que rarement la préservation de l'image du passé ferroviaire et sa médiation au public. Les différentes solutions d'aménagement qui seront proposées doivent dans tous les cas s'appuyer sur la connaissance de ce patrimoine.

J.-F. Belhoste revient pour sa part sur son expérience de vingt années au sein de l'Inventaire général et en particulier sur le rôle de la protection au titre des monuments historiques et de la politique du ministère de la Culture dans l'orientation des recherches et sur l'apport que ces préoccupations patrimoniales ont pu représenter pour la recherche dans le domaine des chemins de fer. Les recherches patrimoniales prenant pour point de départ la culture matérielle sont venues abonder la recherche historique plus large avec par exemple des avancées sur l'histoire des techniques de construction ou sur la notion de réseau, qui inclut désormais les installations qui permettent l'exploitation (comme les ateliers). Cependant, le rapport entre l'histoire des techniques et le patrimoine reste une question difficile, comme le montre le privilège donné aux architectes par rapport aux ingénieurs dans la définition de la valeur patrimoniale d'une œuvre. 
Enfin, l'évolution des institutions chargées de l'étude et de la protection du patrimoine, comme celle des acteurs du secteur ferroviaire, demande l'établissement de nouveaux rapports qui favorisent la recherche.

En conclusion, C. Bergeal souligne combien, pour être vraiment durable et optimal par rapport au territoire, un aménagement nécessite sa connaissance approfondie. La question qui se pose est donc la façon dont nous pouvons aujourd'hui faire reconnaître un patrimoine partagé dans l'aménagement durable de nos territoires.

Round table with Jean-François BELHOSTE, Catherine BERGEAL, Karen BOWIE, Anne HECKER, Evelyne LOHR and François LOYER

Led by Karen Bowie, this discussion allowed each participant to respond to new research proposals from the AHICF academic committee in the areas of railway heritage, architecture, urbanism, and landscape. Grouped around the table were François Loyer, research director at the CNRS, Evelyne Lohr, inventory curator at the Service du patrimoine culturel for the department of Seine-Saint-Denis, Anne Hecker, a geographer and lecturer at the university of Nancy II, JeanFrançois Belhoste, senior researcher and study director at the Ecole pratique des hautes études, and Catherine Bergeal, director of Nature and Landscape at the Ministry of Ecology.

By way of introduction, F. Loyer remarked that far from becoming obsolete as one might have thought in the 1960s, the railway has become the vector of modern mobility. Consequently, the notion of heritage defines itself not simply as nostalgia for a bygone material culture but as a passageway that links two different periods. If cultural heritage and architecture have long been opposed to each other, like urbanism and landscape, similarities are now being noticed that reconstruct these polar notions. As a result, the discu ssion must also include topics such as landscapes in relation to the railway, areas in the vicinity of construction, and design or "architecture without a site."

Each of the participants presented perspectives from their area of research.

Evelyne Lohr analyzed the role of the railway's territorial rights-of-way in the department of Seine-Saint-Denis, which has been affected by very important transformations. She demonstrated how and with which methods of rational and documentary analysis the Service du patrimoine culturel has contributed to an understanding of this complex territory and unified it, interpreting cultural heritage as a force for change and not as an object of nostalgia. Such research can take many directions: for instance, the impact of networks on territory, the railway as a factor of urbanization, the re-use of cultural heritage, and landscapes created by the railway. Such a list presents its own critical problematics, such as which method of study should be applied to heritage of railway lines over a specific parcel of territory, or how to acknowledge a technical heritage that is sometimes difficult to comprehend.

Anne Hecker approaches railway infrastructure, the railway itself, and its hold in terms of cultural heritage. The patrimony of railway lines, epitomized by strongly inflected characteristics (profile, route, innovative methods of construction, a buttress for occupational and local recollections, a conservatory for remarkable flora and fauna) remains unknown. The issue of its partial preservation, however, as a marker and explanation of the evolution of the landscape, must be examined from the double perspective of its valorization through the reopening of routes to traffic or by the transformation of discontinued lines into tourist facilities, bike routes, and nature trails. The latter only rarely assure the preservation of the image of the railway's past and its public mediation. The various planning solutions that will be proposed must be consistently based on knowledge of this cultural heritage.

J.-F. Belhoste returned to his twenty years of experience working closely with the State Inventory Service, particularly addressing the role of protecting historical monuments and the Ministry of Culture's policies regarding research directions and the contribution that patrimonial concerns represent for research in the field of railway studies. Taking material culture as its starting point, 
research on heritage has broadened the scope of historical research with advances in the history of construction techniques or the notion of the network, which from now on must include the installations that enable operations (like workshops). The relationship between the history of techniques and heritage, however, remains a delicate question, as demonstrated by the privilege granted to architects rather than engineers in defining the patrimonial value of a work. Finally, the evolution of the institutions responsible for studying and preserving this heritage, as well as changes that affect those who are involved with the railway sector, demands that new connections be created that favor and support research.

In conclusion, C. Bergeal stressed how important intimate knowledge of a territory is for the longevity and optimal suitability of developmental planning The issue then becomes how we can acknowledge today a heritage that is shared by the long-term developmental planning of our regional landscape.

INDEX

Mots-clés : patrimoine, architecture, historiographie, méthode

Keywords : heritage, historiography, method

\section{AUTEURS}

\section{FRANÇOIS LOYER}

Directeur de recherche au CNRS, Centre André Chastel, Institut national d'histoire de l'art

\section{EVELYNE LOHR}

Conservateur chargée de l'Inventaire, Service du patrimoine culturel, Département de SeineSaint-Denis

\section{ANNE HECKER}

Géographe, maître de conférences à l'université de Nancy II

\section{JEAN-FRANÇOIS BELHOSTE}

Directeur d'études à l'École pratique des hautes études 